\title{
Stability of fractional order fuzzy cellular neural networks with distributed delays via hybrid feedback controllers
}

Ajendra Sing ( $\square$ ajendradu@gmail.com )

Delhi Technological University

Jitendra Nath Rai

Delhi Technological University

\section{Original Research}

Keywords: fractional order, distributed delays, global mittag-letter stability, hybrid feedback controllers, fuzzy cellular neural networks.

Posted Date: February 4th, 2021

DOI: https://doi.org/10.21203/rs.3.rs-170780/v1

License: (c) (i) This work is licensed under a Creative Commons Attribution 4.0 International License.

Read Full License

Version of Record: A version of this preprint was published at Neural Processing Letters on February 23rd, 2021. See the published version at https://doi.org/10.1007/s11063-021-10460-7. 


\title{
Stability of fractional order fuzzy cellular neural networks with distributed delays via hybrid feedback controllers ${ }^{1}$
}

\author{
Ajendra singh ${ }^{a *}$, Jitendra Nath Rai ${ }^{b}$ \\ ${ }^{a}$ Research scholar, Electrical Engineering Department, Delhi Technological University, New \\ Delhi-110042, India \\ ${ }^{b}$ Professor, Electrical Engineering Department, Delhi Technological University, New Delhi -110042, \\ India.
}

\section{Abstract}

This article studies the global Mittag-Leffler stability of fractional order fuzzy cellular neural networks via hybrid feedback controllers. Based on hybrid feedback control technique Lyapunov approach, and some novel analysis techniques of fractional calculation, some sufficient conditions are obtained to guarantee the Global Mittag-lefflers stability. Finally, two simulation example are given to illustrate the effectiveness of the proposed method.

Keywords: fractional order, distributed delays, global mittag-leffler stability, hybrid feedback controllers, fuzzy cellular neural networks.

\section{INTRODUCTION}

Fractional-order calculus have a very long history in pure mathematics. The study of fractional calculus can be dated back to 1695, the fractional operator concept has been put forward by Leibnitz. Fractional calculus as an extension of usual calculus dates from the late seventeenth century and it is regarded as a generalization of derivation and integration of arbitrary order. Over the past few days, the fractional order dynamical system attracts many researchers in different branches, especially science and engineering. Comparing to the traditional integer order dynamical system, the main distinguished influence of fractional order is that infinite memory and more degrees of freedom because it has nonlocal and weakly singular kernels. For the description of memory and hereditary properties of various materials and processes, fractional order models have been proven to be an excellent instrument in comparison with classical integer order models [1]. Actually, the behavior of real world processes generally or most likely is governed by fractional-order systems[2]-[6]. Moreover, fractional-order systems are more likely to be used to describe most of the real-world behaviors than integer-order ones because they can provide more practical value and accurate results. Therefore, many researchers have paid close attention to studying the dynamical behaviors of fractional-order systems and have drawn some wonderful results in the literature [7]-[8].

Stability has been a hot research topic that has drawn much attention from mathematicians, physicists, and computer scientists, and a large amount of results have been available in the literature [9]-[39]. However, most of these results are of integer-order networks. Recently, the various kinds of stability problems for fractional-order differential systems including global stability [40], Exponential stability[41] and Robust stability [42] have been widely discussed. As we all know, the stability problem is a very important performance measure for any dynamical system. [43] investigated the Mittag-Leffler stability of nonlinear fractional neutral singular systems under Caputo and RiemannLiouville derivatives. Mittag-Leffler stability is a relatively new concept related deeply to fractional calculus. In [44], conditions on the Stability of fractional-order nonlinear dynamic systems: Lyapunov direct method and generalized Mittag-Leffler stability are established.

So far, there were three basic CNN structures being proposed. The first one is traditional CNN [45] which is a dynamical and analogical computational network using analog weights, inputs, states and

\footnotetext{
${ }^{1}$ E-mail address: ajendradu@gmail.com

* Corresponding author
} 
outputs. The second one is delay-type CNN [46] which introduces delayed weights into the traditional CNN and uses analog weights, inputs, states and outputs. The last one is discrete-time CNN [47] which uses analog weights, inputs, states, and digital outputs. Unlike the traditional CNNs structure, FCNNs implements fuzzy logic between its template input and/or output besides the sum of product operations. Different from the cellular neural networks, fuzzy cellular neural networks integrate fuzzy logic into the structure of customary cell neural systems and keeps up nearby connectedness among cells. Fuzzy cellular neural networks (FCNNs), introduced by Yang et al. [48]-[49]. such as thinking and reasoning, [50], [51] white blood cell detection, [52] and pattern recognition. On the other hand, outer synchronization between two coupled networks has been extensively studied. Similar to the traditional CNNs, the stability of the system is very important in the design of FCNNs. We note that several conditions for FCNNs have been proposed [53]-[54].

Between multiple performance objectives using a single feedback function, whereas hybrid control seeks to achieve multiple performance objectives in a locally adaptive sense by exchanging between individuals from a from the earlier determined group of input capacities. There are numerous outcomes on half breed frameworks and cross breed control in the distributed writing. Conferences have been held with published proceedings [55]-[56], and special issues of archival publications have been devoted to hybrid systems and control [57] - [61]. In addition, the literature on hybrid control systems is very large; a number of references to this literature are identified throughout this paper. Tutorial papers [62]-[64], a monograph [65], and doctoral dissertations that treat hybrid control systems have been written. Examples of hybrid controllers in the automotive field include applications for automated highway systems [66], motion planning [67]-[69], and so on. Despite the fact that, examine in half breed control has been the article of an extraordinary and gainful exertion in the ongoing years in the car field, from the best of our knowledge, this is the first time that benefits of both FOC and hybrid control are used for ACC maneuvers.

Motivated by the above discussions, the objective of this paper is to study the global Mittag-Leffler stability of fractional order fuzzy cellular neural networks with distributed delays via hybrid feedback controllers. To the best of authors knowledge global Mittag-Leffler stability of fractional order fuzzy cellular neural networks with distributed delays via hybrid feedback controllers is not yet fully studied. By constructing a suitable Lyapunov functional, Stability criteria are derived to ensure the stability of concerned fractional order systems. Caputo definition are applied. An effective controller is achieved for synchronization of master and slave systems considered. A numerical examples is illustrated to show the correctness of proposed method.

The novelties of the study are given below:

- We studied the global Mittag-Leffler stability of fractional order fuzzy cellular neural networks.

- Constructed a novel Lyapunov functional for check the stabilty of the fractional order system.

- Stability criteria are derived to ensure the stability of concerned fractional order systems.

- An effective hybrid feedback controller is applied for synchronization of master and slave systems.

The paper is organized as follows. In section 3, model description of fractional order neural networks and preliminaries are presented. In section 4, main results are drawn. In section 5, numerical example is performed to verify the effectiveness of our method. Finally, conclusion results are drawn.

\section{MODEL DESCRIPTION AND PRELIMINARIES}

In the present section, we introduce some basic definitions and the corresponding results will be used later. 
Definition 1.([70]) Suppose that $\beta>0$ for a function $f(t)$ is defined as

$$
J^{\beta} f(t)=\frac{1}{\Gamma(\beta)} \int_{b}^{t}(t-\tau)^{\beta-1} f(\tau) d \tau,
$$

where $t \geq b, t \in \mathbb{R}, \Gamma(\cdot)$ is the gamma function, given by $\Gamma(\beta)=\int_{0}^{\infty} t^{s-1} e^{-t} d t$.

Definition 2.([70]) The Caputo fractional derivative of order $\beta$ for a function $f(t)$ is defined as

$$
D^{\beta} f(t)=\frac{1}{\Gamma(n-\beta)} \int_{b}^{t}(t-\tau)^{n-\beta-1} f^{(n)}(\tau) d \tau,
$$

in which $t \geq b, t \in \mathbb{R}, n-1<\beta<n \in \mathbb{N}$.

Definition 3([71]). The one-parameter Mittag-Leffler function is defined as

$$
E_{\phi}(x)=\sum_{l=0}^{\infty} \frac{x^{l}}{\gamma(l \phi+1)} .
$$

Definition 4([71]). The two-parameter Mittag-Leffler function is defined as

$$
E_{\phi, \psi}(x)=\sum_{l=0}^{\infty} \frac{x^{l}}{\gamma(l \phi+\psi)} .
$$

Definition 5([71]). Moreover, the Laplace transform of Mittag-Leffler function in two parameters is

$$
\mathbb{L}\left\{t^{\psi-1} E_{\phi, \psi}\left(-\lambda t^{\phi}\right)\right\}=\frac{s^{\phi-\psi}}{s^{\phi}+\lambda},\left(R(s)>|\lambda|^{\frac{1}{\phi}}\right),
$$

where $\mathrm{s}$ is the variable in Laplace domain, $R(s)$ denotes the real part of $\mathrm{s}, \lambda \in R$ and $L$. stands for the Laplace transform.

Lemma $1([72])$.Let a vector-value function $p(t) \subset \mathbb{R}^{n}$ is differentiable. Then, for any $t>0$, one has

$$
\frac{1}{2} D^{\beta} p^{2}(t) \leq p(t) D^{\beta} p(t)
$$

when $0<\beta<1$.

Lemma 2([73]). Suppose that $x, y \in \mathbb{R}^{n}$ be the two states of the system (1). Then, one has

$$
\begin{aligned}
& \left|\bigwedge_{j=1}^{n} u_{i j} f_{j}\left(\vartheta_{j}\right)-\bigwedge_{j=1}^{n} u_{i j} f_{j}\left(\zeta_{j}\right)\right| \leq \sum_{j=1}^{n}\left|u_{i j}\right|\left|f_{j}\left(\vartheta_{j}\right)-f_{j}\left(\zeta_{j}\right)\right|, \\
& \left|\bigvee_{j=1}^{n} v_{i j} f_{j}\left(\vartheta_{j}\right)-\bigvee_{j=1}^{n} v_{i j} f_{j}\left(\zeta_{j}\right)\right| \leq \sum_{j=1}^{n}\left|v_{i j}\right|\left|f_{j}\left(\vartheta_{j}\right)-f_{j}\left(\zeta_{j}\right)\right| .
\end{aligned}
$$

Lemma $3([74])$. Let $k_{1}>0, k_{2}>0, k_{3}>0, k_{4}>1$, and $\left(\frac{1}{k}{ }_{3}\right)+\left(\frac{1}{k}{ }_{4}\right)=1$. Then for any $\epsilon>0$, we have

$$
k_{1} k_{2} \leq \frac{1}{k_{3}}\left(k_{1} \epsilon\right)^{k_{3}}+\frac{1}{k_{4}}\left(k_{2} \frac{1}{\epsilon}\right)^{k_{4}}
$$

The equality holds if and only if $\left(k_{1} \epsilon\right)^{k_{3}}=\left(k_{2}\left(\frac{1}{\epsilon}\right)\right)^{k_{4}}$.

Lemma $4([75])$. Let $\mathrm{V}(\mathrm{t})$ be a continuous function on $[0, \infty)$ satisfying

$$
{ }^{C} D^{\alpha} V(t) \leq-\gamma V(t), \alpha \in(0,1),
$$

for some constant $\gamma$. Then

$$
V(t) \leq V(0) E_{\alpha}\left(-\gamma t^{\alpha}\right), t \geq 0
$$

Lemma $5([76])$. Let $u>0, v>0,1<p<+\infty$, and $\frac{1}{p}+\frac{1}{q}=1$, then the following relationship holds

$$
u v \leq \frac{1}{p} u^{p}+\frac{1}{q} v^{q},
$$


the equality holds if and only if $u=v^{p-1}$.

Assumption 1. The neuron activation functions $f_{j}$ are Lipschitz continuous and satisfy for

$$
\left|f_{j}\left(\vartheta_{j}\right)-f_{j}\left(\zeta_{j}\right)\right| \leq F_{j}\left|\vartheta_{j}-\zeta_{j}\right|
$$

positive constants $F_{j}>0$.

\section{Main Results}

In this section, we will derive some criteria to guarantee the Global Mittag-Leffler stability between drive system (10) and response system (11).

In this paper, we consider the following n-dimensional FCNNs with distributed delay:

$$
\begin{aligned}
{ }^{C} D^{\alpha} \zeta_{i}(t) & =-d_{i} \zeta_{i}(t)+\sum_{j=1}^{n} c_{i j} f_{j}\left(\zeta_{j}(t)\right)+\sum_{j=1}^{n} a_{i j} f_{j}\left(\zeta_{j}(t-\tau)\right)+\sum_{j=1}^{n} h_{i j} \mu_{j} \\
& +\bigwedge_{j=1}^{n} u_{i j} \int_{0}^{\infty} k_{j}(s) f_{j}\left(\zeta_{j}(s-\tau)\right) d s+\bigwedge_{j=1}^{n} P_{i j} \mu_{j}+\bigvee_{j=1}^{n} v_{i j} \int_{0}^{\infty} k_{j}(s) f_{j}\left(\zeta_{j}(s-\tau)\right) d s \\
& +\bigvee_{j=1}^{n} Q_{i j} \mu_{j}+I_{i}, i=1,2, \ldots, n
\end{aligned}
$$

where, ${ }^{C} D^{\alpha}$ is the Caputo's fractional derivative and $0<\alpha<1, d_{i}>0$ denote the rate with which the ith neuron, $\zeta_{i}(t)$ corresponds to the state variable of the ith unit at time $\mathrm{t}, c_{i j}, a_{i j}$ are elements of feedback and feed forward template $h_{i j}$ is the feed-forward template. $u_{i j}, v_{i j}, P_{i j}, Q_{i j}$ are elements of fuzzy feedback MIN template, fuzzy feedback MAX template, respectively. $\bigvee$ and $\wedge$ denote the fuzzy AND and fuzzy OR operation, respectively. $I_{i}$ denote state, input and bias of the ith neuron, respectively. $f_{i}$ is the activation function.

In this paper, we refer to model (10) as the drive system, the response system is given as follows

$$
\begin{aligned}
{ }^{C} D^{\alpha} \vartheta_{i}(t) & =-d_{i} \vartheta_{i}(t)+\sum_{j=1}^{n} c_{i j} f_{j}\left(\vartheta_{j}(t)\right)+\sum_{j=1}^{n} a_{i j} f_{j}\left(\vartheta_{j}(t-\tau)\right)+\sum_{j=1}^{n} h_{i j} \mu_{j} \\
& +\bigwedge_{j=1}^{n} u_{i j} \int_{0}^{\infty} k_{j}(s) f_{j}\left(\vartheta_{j}(s-\tau)\right) d s+\bigwedge_{j=1}^{n} P_{i j} \mu_{j}+\bigvee_{j=1}^{n} v_{i j} \int_{0}^{\infty} k_{j}(s) f_{j}\left(\vartheta_{j}(s-\tau)\right) d s \\
& +\bigvee_{j=1}^{n} Q_{i j} \mu_{j}+I_{i}+u_{i}(t),
\end{aligned}
$$

where $\vartheta_{i}(t)$ corresponds to the state variable of the ith neuron of the response system and $u_{i}(t)$ indicates the control input.

Suppose that $\zeta_{i}(t), \vartheta_{i}(t)$ are arbitrary solutions of system (10) and system (11) respectively, and let $e_{i}(t)=\vartheta_{i}(t)-\zeta_{i}(t)$ for $i \in I$, then from system (10) and system (11), the error dynamical system can be derived as

$$
\begin{aligned}
\xi_{i}(t) & \left.=-d_{i} \xi_{i}(t)+\sum_{j=1}^{n} c_{i j}\left[f_{j}\left(\vartheta_{j}(t)\right)-f_{j}\left(\zeta_{j}(t)\right)\right]+\sum_{j=1}^{n} a_{i j}\left[f_{j}\left(\vartheta_{j}(t-\tau)\right)\right)-f_{j}\left(\zeta_{j}(t-\tau)\right)\right] \\
& +\bigwedge_{j=1}^{n} u_{i j} \int_{0}^{\infty} k_{j}(s)\left[f_{j}\left(\vartheta_{j}(t-\tau)\right)-f_{j}\left(\zeta_{j}(t-\tau)\right)\right] d s \\
& +\bigvee_{j=1}^{n} v_{i j} \int_{0}^{\infty} k_{j}(s)\left[f_{j}\left(\vartheta_{j}(t-\tau)\right)-f_{j}\left(\zeta_{j}(t-\tau)\right)\right] d s+u_{i}(t)
\end{aligned}
$$


Now we introduce the hybrid feedback controls are

$u_{i}(t)=u_{1 i}(t)+u_{2 i}(t)$, where $u_{1 i}(t)=-\phi_{i}(t) \xi_{i}(t)$ and $u_{2 i}(t)=-\Psi_{i}(t) \xi_{i}(t)$.

$$
\begin{aligned}
\xi_{i}(t) & =-d_{i} \xi_{i}(t)+\sum_{j=1}^{n} c_{i j}\left[f_{j}\left(\vartheta_{j}(t)\right)-f_{j}\left(\zeta_{j}(t)\right)\right]+\sum_{j=1}^{n} a_{i j}\left[f_{j}\left(\vartheta_{j}(t-\tau)\right)-f_{j}\left(\zeta_{j}(t-\tau)\right)\right] \\
& +\bigwedge_{j=1}^{n} u_{i j} \int_{0}^{\infty} k_{j}(s)\left[f_{j}\left(\vartheta_{j}(t-\tau)\right)-f_{j}\left(\zeta_{j}(t-\tau)\right)\right] d s \\
& +\bigvee_{j=1}^{n} v_{i j} \int_{0}^{\infty} k_{j}(s)\left[f_{j}\left(\vartheta_{j}(t-\tau)\right)-f_{j}\left(\zeta_{j}(t-\tau)\right)\right] d s-\phi_{i}(t) \xi_{i}(t)-\Psi_{i}(t) \xi_{i}(t)
\end{aligned}
$$

Remark 4.1. Motivated by the success of the applications of the Mittag-Leffler functions in many areas of science and engineering, we present our work here. Applications of Mittag-Leffler functions in certain areas of physical and applied sciences are also very common. Very recently Chen et al. [77] formulated and investigated the global Mittag-Leffler stability and synchronization of fractionalorder memristor NNs. Stamova and Stamov [78] studied the impulsive and linear controllers on Mittag-Leffler synchronization of fractional-order NNs with time-varying delays. For extending the application of fractional calculus in nonlinear systems, we propose the Mittag-Leffler stability and the Lyapunov direct method with a view to enrich the knowledge of both system theory and fractional calculus.

Theorem 4.2. Assume that Assumption (1) and Lemma (4) hold, then the system (10) is Globally mittag-leffler stable, provided the following conditions holds:

$$
\begin{aligned}
\eta_{1}(t) & >\eta_{2}(t), \\
\eta_{1}(t) & =\min _{1 \leq i \leq}\left[\left(d_{i}+\phi_{i}(t)+\Psi_{i}(t)\right)-\sum_{j=1}^{n} c_{i j} F_{j} \rho^{-1}-\sum_{j=1}^{n} c_{j i} F_{i} \rho-\sum_{j=1}^{n} a_{i j} F_{j} \rho^{-1}\right. \\
& \left.+\sum_{j=1}^{n}\left|u_{i j}\right| F_{j} \rho^{-1}+\sum_{j=1}^{n}\left|v_{i j}\right| F_{j} \rho^{-1}\right]>0 \\
\eta_{2}(t) & =\max _{1 \leq i \leq n}\left[\sum_{j=1}^{n} a_{j i} F_{i} \rho+\sum_{j=1}^{n}\left|u_{j i}\right| F_{i} \rho+\sum_{j=1}^{n}\left|v_{j i}\right| F_{i} \rho\right]>0
\end{aligned}
$$

Proof: Let us consider the Lyapunov function is

$$
V(t)=\sum_{i=1}^{n} \frac{1}{2} \xi_{i}^{2}(t)
$$

According to the Caputo fractional-order derivative definition, we have

$$
\begin{aligned}
{ }^{C} D^{\alpha} V(t, \xi(t)) & \leq \sum_{i=1}^{n} \xi_{i}(t)^{C} D^{\alpha} \xi_{i}(t) \\
& \leq \sum_{i=1}^{n} \xi_{i}(t)\left[-d_{i} \xi_{i}(t)+\sum_{j=1}^{n} c_{i j}\left[f_{j}\left(\vartheta_{j}(t)\right)-f_{j}\left(\zeta_{j}(t)\right)\right]\right. \\
& +\sum_{j=1}^{n} a_{i j}\left[f_{j}\left(\vartheta_{j}(t-\tau)\right)-f_{j}\left(\zeta_{j}(t-\tau)\right)\right] \\
& +\bigwedge_{j=1}^{n}\left|u_{i j}\right| \int_{0}^{\infty} k_{j}(s)\left[f_{j}\left(\vartheta_{j}(t-\tau)\right)-f_{j}\left(\zeta_{j}(t-\tau)\right)\right] d s
\end{aligned}
$$




$$
\begin{aligned}
& \left.+\bigvee_{j=1}^{n}\left|v_{i j}\right| \int_{0}^{\infty} k_{j}(s)\left[f_{j}\left(\vartheta_{j}(t-\tau)\right)-f_{j}\left(\zeta_{j}(t-\tau)\right)\right] d s-\phi_{i}(t) \xi_{i}(t)-\Psi_{i}(t) \xi_{i}(t)\right] \\
& =-\sum_{i=1}^{n} d_{i} \xi_{i}^{2}(t)+\sum_{i=1}^{n} \sum_{j=1}^{n} c_{i j} F_{j}\left|\xi_{i}(t)\right|\left|\xi_{j}(t)\right|+\sum_{i=1}^{n} \sum_{j=1}^{n} a_{i j} F_{j}\left|\xi_{i}(t)\right|\left|\xi_{j}(t-\tau)\right| \\
& +\sum_{i=1}^{n} \sum_{j=1}^{n}\left|u_{i j}\right| \int_{0}^{\infty} k_{j}(s) F_{j}\left|\xi_{i}(s)\right|\left|\xi_{j}(s-\tau)\right| d s \\
& +\sum_{i=1}^{n} \sum_{j=1}^{n}\left|v_{i j}\right| \int_{0}^{\infty} k_{j}(s) F_{j}\left|\xi_{i}(s)\right|\left|\xi_{j}(s-\tau)\right| d s-\sum_{i=1}^{n} \phi_{i}(t) \xi_{i}^{2}(t)-\sum_{i=1}^{n} \Psi_{i}(t) \xi_{i}^{2}(t) \\
& \leq-\sum_{i=1}^{n} d_{i} \xi_{i}^{2}(t)+\sum_{i=1}^{n} \sum_{j=1}^{n} c_{i j} F_{j}\left[\frac{\rho^{-1}}{2} \xi_{i}^{2}(t)+\frac{\rho}{2} \xi_{j}^{2}(t)\right] \\
& +\sum_{i=1}^{n} \sum_{j=1}^{n} a_{i j} F_{j}\left[\frac{\rho^{-1}}{2} \xi_{i}^{2}(t)+\frac{\rho}{2} \xi_{j}^{2}(t-\tau)\right] \\
& +\sum_{i=1}^{n} \sum_{j=1}^{n}\left|u_{i j}\right| \int_{0}^{\infty} k_{j}(s) F_{j}\left[\frac{\rho^{-1}}{2} \xi_{i}^{2}(s)+\frac{\rho}{2} \xi_{j}^{2}(s-\tau)\right] d s \\
& +\sum_{i=1}^{n} \sum_{j=1}^{n}\left|v_{i j}\right| \int_{0}^{\infty} k_{j}(s) F_{j}\left[\frac{\rho^{-1}}{2} \xi_{i}^{2}(s)+\frac{\rho}{2} \xi_{j}^{2}(s-\tau)\right] d s-\sum_{i=1}^{n} \phi_{i}(t) \xi_{i}^{2}(t)-\sum_{i=1}^{n} \Psi_{i}(t) \xi_{i}^{2}(t) \\
& \leq-\sum_{i=1}^{n} d_{i} \xi_{i}^{2}(t)+\sum_{i=1}^{n} \sum_{j=1}^{n} c_{i j} F_{j}\left[\frac{\rho^{-1}}{2} \xi_{i}^{2}(t)\right]+\sum_{i=1}^{n} \sum_{j=1}^{n} c_{i j} F_{j}\left[\frac{\rho}{2} \xi_{j}^{2}(t)\right] \\
& +\sum_{i=1}^{n} \sum_{j=1}^{n} a_{i j} F_{j}\left[\frac{\rho^{-1}}{2} \xi_{i}^{2}(t)\right]+\sum_{i=1}^{n} \sum_{j=1}^{n} a_{i j} F_{j}\left[\frac{\rho}{2} \xi_{j}^{2}(t-\tau)\right] \\
& +\sum_{i=1}^{n} \sum_{j=1}^{n}\left|u_{i j}\right| \int_{0}^{\infty} k_{j}(s) F_{j}\left[\frac{\rho^{-1}}{2} \xi_{i}^{2}(s)\right] d s+\sum_{i=1}^{n} \sum_{j=1}^{n}\left|u_{i j}\right| \int_{0}^{\infty} k_{j}(s) F_{j}\left[\frac{\rho}{2} \xi_{j}^{2}(s-\tau)\right] d s \\
& +\sum_{i=1}^{n} \sum_{j=1}^{n}\left|v_{i j}\right| \int_{0}^{\infty} k_{j}(s) F_{j}\left[\frac{\rho^{-1}}{2} \xi_{i}^{2}(s)\right] d s+\sum_{i=1}^{n} \sum_{j=1}^{n}\left|v_{i j}\right| \int_{0}^{\infty} k_{j}(s) F_{j}\left[\frac{\rho}{2} \xi_{j}^{2}(s-\tau)\right] d s \\
& -\sum_{i=1}^{n} \phi_{i}(t) \xi_{i}^{2}(t)-\sum_{i=1}^{n} \Psi_{i}(t) \xi_{i}^{2}(t) \\
& \leq-\sum_{i=1}^{n} d_{i} \xi_{i}^{2}(t)+\sum_{i=1}^{n} \sum_{j=1}^{n} c_{i j} F_{j}\left[\frac{\rho^{-1}}{2} \xi_{i}^{2}(t)\right]+\sum_{i=1}^{n} \sum_{j=1}^{n} c_{j i} F_{i}\left[\frac{\rho}{2} \xi_{i}^{2}(t)\right] \\
& +\sum_{i=1}^{n} \sum_{j=1}^{n} a_{i j} F_{j}\left[\frac{\rho^{-1}}{2} \xi_{i}^{2}(t)\right]+\sum_{i=1}^{n} \sum_{j=1}^{n} a_{j i} F_{i}\left[\frac{\rho}{2} \xi_{i}^{2}(t-\tau)\right] \\
& +\sum_{i=1}^{n} \sum_{j=1}^{n}\left|u_{i j}\right| \int_{0}^{\infty} k_{j}(s) F_{j}\left[\frac{\rho^{-1}}{2} \xi_{i}^{2}(s)\right] d s+\sum_{i=1}^{n} \sum_{j=1}^{n}\left|u_{j i}\right| \int_{0}^{\infty} k_{j}(s) F_{i}\left[\frac{\rho}{2} \xi_{i}^{2}(s-\tau)\right] d s \\
& +\sum_{i=1}^{n} \sum_{j=1}^{n}\left|v_{i j}\right| \int_{0}^{\infty} k_{j}(s) F_{j}\left[\frac{\rho^{-1}}{2} \xi_{i}^{2}(s)\right] d s+\sum_{i=1}^{n} \sum_{j=1}^{n}\left|v_{j i}\right| \int_{0}^{\infty} k_{j}(s) F_{i}\left[\frac{\rho}{2} \xi_{i}^{2}(s-\tau)\right] d s \\
& -\sum_{i=1}^{n} \phi_{i}(t) \xi_{i}^{2}(t)-\sum_{i=1}^{n} \Psi_{i}(t) \xi_{i}^{2}(t)
\end{aligned}
$$




$$
\begin{aligned}
& \leq \sum_{i=1}^{n}\left[-\left(d_{i}+\phi_{i}(t)+\Psi_{i}(t)\right)+\sum_{j=1}^{n} c_{i j} F_{j} \frac{\rho^{-1}}{2}+\sum_{j=1}^{n} c_{j i} F_{i} \frac{\rho}{2}+\sum_{j=1}^{n} a_{i j} F_{j}\left[\frac{\rho^{-1}}{2}\right]\right] \xi_{i}^{2}(t) \\
& +\sum_{i=1}^{n}\left[\sum_{j=1}^{n} a_{j i} F_{i}\left[\frac{\rho}{2}\right]\right] \xi_{i}^{2}(t-\tau)+\sum_{i=1}^{n}\left[\sum_{j=1}^{n}\left|u_{i j}\right| \int_{0}^{\infty} k_{j}(s) F_{j}\left[\frac{\rho^{-1}}{2}\right] d s\right. \\
& \left.+\sum_{j=1}^{n}\left|v_{i j}\right| \int_{0}^{\infty} k_{j}(s) F_{j}\left[\frac{\rho^{-1}}{2}\right]\right] \xi_{i}^{2}(s) d s+\sum_{i=1}^{n}\left[\sum_{j=1}^{n}\left|u_{j i}\right| \int_{0}^{\infty} k_{j}(s) F_{i}\left[\frac{\rho}{2}\right] d s\right. \\
& \left.+\sum_{j=1}^{n}\left|v_{j i}\right| \int_{0}^{\infty} k_{j}(s) F_{i}\left[\frac{\rho}{2}\right]\right] \xi_{i}^{2}(s-\tau) d s \\
& \leq-\sum_{i=1}^{n}\left[\left(d_{i}+\phi_{i}(t)+\Psi_{i}(t)\right)-\sum_{j=1}^{n} c_{i j} F_{j} \frac{\rho^{-1}}{2}-\sum_{j=1}^{n} c_{j i} F_{i} \frac{\rho}{2}-\sum_{j=1}^{n} a_{i j} F_{j}\left[\frac{\rho^{-1}}{2}\right]\right] \xi_{i}^{2}(t) \\
& +\sum_{i=1}^{n}\left[\sum_{j=1}^{n} a_{j i} F_{i}\left[\frac{\rho}{2}\right]\right] \xi_{i}^{2}(t-\tau)+\sum_{i=1}^{n}\left[\sum_{j=1}^{n}\left|u_{i j}\right| \int_{0}^{\infty} k_{j}(s) F_{j}\left[\frac{\rho^{-1}}{2}\right] d s\right. \\
& \left.+\sum_{j=1}^{n}\left|v_{i j}\right| \int_{0}^{\infty} k_{j}(s) F_{j}\left[\frac{\rho^{-1}}{2}\right]\right] \xi_{i}^{2}(s) d s+\sum_{i=1}^{n}\left[\sum_{j=1}^{n}\left|u_{j i}\right| \int_{0}^{\infty} k_{j}(s) F_{i}\left[\frac{\rho}{2}\right] d s\right. \\
& \left.+\sum_{j=1}^{n}\left|v_{j i}\right| \int_{0}^{\infty} k_{j}(s) F_{i}\left[\frac{\rho}{2}\right]\right] \xi_{i}^{2}(s-\tau)(s) d s \\
& \leq-\eta_{1} V(t, \xi(t))+\eta_{2} \max _{t-\tau \leq s \leq t} V(s, \xi(s)),
\end{aligned}
$$

where,

$$
\begin{aligned}
\eta_{1}(t) & =\min _{1 \leq i \leq n}\left[\left(d_{i}+\phi_{i}(t)+\Psi_{i}(t)\right)-\sum_{j=1}^{n} c_{i j} F_{j} \rho^{-1}-\sum_{j=1}^{n} c_{j i} F_{i} \rho-\sum_{j=1}^{n} a_{i j} F_{j} \rho^{-1}\right. \\
& \left.+\sum_{j=1}^{n}\left|u_{i j}\right| F_{j} \rho^{-1}+\sum_{j=1}^{n}\left|v_{i j}\right| F_{j} \rho^{-1}\right]>0 \\
\eta_{2}(t) & =\max _{1 \leq i \leq n}\left[\sum_{j=1}^{n} a_{j i} F_{i} \rho+\sum_{j=1}^{n}\left|u_{j i}\right| F_{i} \rho+\sum_{j=1}^{n}\left|v_{j i}\right| F_{i} \rho\right]>0 .
\end{aligned}
$$

From this estimate, for any solution (13), which satisfies the Razumichin condition

$$
V(s, \xi(s)) \leq V(t, \xi(t)), t-\tau \leq s \leq t,
$$

we have

$$
{ }^{C} D^{\alpha} V(t, \xi(t)) \leq-\left(\eta_{1}-\eta_{2}\right) V(t, \xi(t)), t \geq 0 .
$$

According to Lemma 5, the claim of Theorem 4.1 follows.

$$
V(t, \xi(t)) \leq V(0, \xi(0)) E_{\alpha}\left(-\gamma t^{\alpha}\right), t \geq 0
$$

By Lemma 4 and inequality (19), this proves system (10) can be achieved globally Mittag-Leffler stabilization under the designed control law.

Hence complete the proof.

Theorem 4.3. Assume that Assumption (1) and Lemma (4) hold, then the system (10) is Globally mittag-leffler stable, provided the following conditions holds:

$$
\theta_{1}(t)>\theta_{2}(t)
$$




$$
\begin{aligned}
\theta_{1}(t) & =\min _{1 \leq i \leq n}\left[\omega\left(d_{i}+\left|\phi_{i}(t)\right|+\left|\Psi_{i}(t)\right|\right)-\sum_{j=1}^{n}\left|c_{i j}\right| F_{j} \delta_{1}(\omega-1)-\sum_{j=1}^{n}\left|c_{j i}\right| F_{i} \frac{1}{\delta_{1}^{\omega-1}}\right. \\
& \left.-\sum_{j=1}^{n}\left|a_{i j}\right| F_{j}(\omega-1) \delta_{2}+\left|u_{i j}\right| F_{j}(\omega-1) \delta_{3}+\left|v_{i j}\right| F_{j}(\omega-1) \delta_{3}\right] \frac{\left|\xi_{i}(t)\right|^{\omega}}{\omega}>0, \\
\theta_{2}(t) & =\max _{1 \leq i \leq n}\left[\sum_{j=1}^{n}\left|a_{j i}\right| F_{i} \frac{1}{\delta_{2}^{\omega-1}}+\sum_{j=1}^{n}\left|u_{j i}\right| F_{i} \frac{1}{\delta_{3}^{\omega-1}}+\sum_{j=1}^{n}\left|v_{j i}\right| F_{i} \frac{1}{\delta_{3}^{\omega-1}}\right] \frac{\left|\xi_{i}(t-\tau)\right|^{\omega}}{\omega}>0 .
\end{aligned}
$$

Proof: we define a Lyapunov function

$$
V(t, \xi(t))=\sum_{i=1}^{n} \frac{1}{\omega}\left|\xi_{i}(t)\right|^{\omega}
$$

According to the Caputo fractional-order derivative definition, we have

$$
\begin{aligned}
{ }^{C} D^{\alpha} V(t, \xi(t)) \leq & \sum_{i=1}^{n}\left|\xi_{i}(t)\right|^{\omega-1}\left[-d_{i}\left|\xi_{i}(t)\right|+\sum_{j=1}^{n}\left|c_{i j}\right|\left[f_{j}\left(\vartheta_{j}(t)\right)-f_{j}\left(\zeta_{j}(t)\right)\right]\right. \\
& +\sum_{j=1}^{n}\left|a_{i j}\right|\left[f_{j}\left(\vartheta_{j}(t-\tau)\right)-f_{j}\left(\zeta_{j}(t-\tau)\right)\right] \\
& +\bigwedge_{j=1}^{n}\left|u_{i j}\right| \int_{0}^{\infty} k_{j}(s)\left[f_{j}\left(\vartheta_{j}(s-\tau)\right)-f_{j}\left(\zeta_{j}(t-\tau)\right)\right] d s \\
& \left.+\bigvee_{j=1}^{n}\left|v_{i j}\right| \int_{0}^{\infty} k_{j}(s)\left[f_{j}\left(\vartheta_{j}(s-\tau)\right)-f_{j}\left(\zeta_{j}(t-\tau)\right)\right] d s+\left|\phi_{i}(t)\right|\left|\xi_{i}(t)\right|+\left|\Psi_{i}(t)\right|\left|\xi_{i}(t)\right|\right] \\
& \leq \sum_{i=1}^{n}\left|\xi_{i}(t)\right|^{\omega-1}\left[-d_{i}\left|\xi_{i}(t)\right|+\sum_{j=1}^{n}\left|c_{i j}\right| F_{j}\left|\xi_{j}(t)\right|+\sum_{j=1}^{n}\left|a_{i j}\right| F_{j}\left|\xi_{j}(t-\tau)\right|\right. \\
& +\sum_{j=1}^{n}\left|u_{i j}\right| \int_{0}^{\infty} k_{j}(s) F_{j}\left|\xi_{j}(s-\tau)\right| d s+\sum_{j=1}^{n}\left|v_{i j}\right| \int_{0}^{\infty} k_{j}(s) F_{j}\left|\xi_{j}(s-\tau)\right| d s \\
& \left.+\left|\phi_{i}(t)\right|\left|\xi_{i}(t)\right|+\left|\Psi_{i}(t)\right|\left|\xi_{i}(t)\right|\right] \\
& \leq-\sum_{i=1}^{n}\left(d_{i}+\left|\phi_{i}(t)\right|+\left|\Psi_{i}(t)\right|\right)\left|\xi_{i}(t)\right|^{\omega}+\sum_{i=1}^{n} \sum_{j=1}^{n}\left|c_{i j}\right| F_{j}\left|\xi_{i}(t)\right|^{\omega-1}\left|\xi_{j}(t)\right| \\
& +\sum_{i=1}^{n} \sum_{j=1}^{n}\left|a_{i j}\right| F_{j}\left|\xi_{i}(t)\right|^{\omega-1}\left|\xi_{j}(t-\tau)\right|+\sum_{i=1}^{n} \sum_{j=1}^{n}\left|u_{i j}\right| \int_{0}^{\infty} k_{j}(s) F_{j}\left|\xi_{i}(s)\right|^{\omega-1}\left|\xi_{j}(s-\tau)\right| d s \\
& +\sum_{i=1}^{n} \sum_{j=1}^{n}\left|v_{i j}\right| \int_{0}^{\infty} k_{j}(s) F_{j}\left|\xi_{i}(s)\right|^{\omega-1}\left|\xi_{j}(s-\tau)\right| d s
\end{aligned}
$$

From Lemma 3 we have

$$
\begin{aligned}
\left|\xi_{i}(t)\right|^{\omega-1}\left|\xi_{j}(t)\right| & \leq \frac{\omega-1}{\omega}\left[\left|\xi_{i}(t)\right|^{\omega-1} \delta_{1}^{\frac{\omega-1}{\omega}}\right]^{\frac{\omega}{\omega-1}}+\frac{1}{\omega}\left[\left|\xi_{j}(t)\right| \delta_{1}^{\frac{-\omega-1}{\omega}}\right]^{\omega} \\
& =\frac{\omega-1}{\omega}\left|\xi_{i}(t)\right|^{\omega} \delta_{1}+\frac{1}{\omega}\left|\xi_{j}(t)\right|^{\omega} \frac{1}{\delta_{1}^{\omega-1}} \\
\left|\xi_{i}(t)\right|^{\omega-1}\left|\xi_{j}(t-\tau)\right| & \leq \frac{\omega-1}{\omega}\left[\left|\xi_{i}(t)\right|^{\omega-1} \delta_{2}^{\frac{\omega-1}{\omega}}\right]^{\frac{\omega}{\omega-1}}+\frac{1}{\omega}\left[\left|\xi_{j}(t-\tau)\right| \delta_{2}^{\frac{-\omega-1}{\omega}}\right]^{\omega}
\end{aligned}
$$




$$
\begin{aligned}
& =\frac{\omega-1}{\omega}\left|\xi_{i}(t)\right|^{\omega} \delta_{2}+\frac{1}{\omega}\left|\xi_{j}(t-\tau)\right|^{\omega} \frac{1}{\delta_{2}^{\omega-1}} \\
\int_{0}^{\infty}\left|\xi_{i}(s)\right|^{\omega-1}\left|\xi_{j}(s-\tau)\right| d s & \leq \int_{0}^{\infty} \frac{\omega-1}{\omega}\left[\left|\xi_{i}(s)\right|^{\omega-1} \delta_{3}^{\frac{\omega-1}{\omega}}\right]^{\frac{\omega}{\omega-1}}+\frac{1}{\omega}\left[\left|\xi_{j}(s-\tau)\right| \delta_{3}^{\frac{-\omega-1}{\omega}}\right]^{\omega} \\
& =\int_{0}^{\infty} \frac{\omega-1}{\omega}\left|\xi_{i}(s)\right|^{\omega} \delta_{3}+\frac{1}{\omega}\left|\xi_{j}(s-\tau)\right|^{\omega} \frac{1}{\delta_{3}^{\omega-1}}
\end{aligned}
$$

Now substitute the above values in the above equation we get

$$
\begin{aligned}
& { }^{C} D^{\alpha} V(t, \xi(t)) \leq-\sum_{i=1}^{n}\left(d_{i}+\left|\phi_{i}(t)\right|+\left|\Psi_{i}(t)\right|\right)\left|\xi_{i}(t)\right|^{\omega}+\sum_{i=1}^{n} \sum_{j=1}^{n}\left|c_{i j}\right| F_{j}\left[\frac{\omega-1}{\omega}\left|\xi_{i}(t)\right|^{\omega} \delta_{1}\right. \\
& \left.+\frac{1}{\omega}\left|\xi_{j}(t)\right|^{\omega} \frac{1}{\delta_{1}^{\omega-1}}\right]+\sum_{i=1}^{n} \sum_{j=1}^{n}\left|a_{i j}\right| F_{j}\left[\frac{\omega-1}{\omega}\left|\xi_{i}(t)\right|^{\omega} \delta_{2}+\frac{1}{\omega}\left|\xi_{j}(t-\tau)\right|^{\omega} \frac{1}{\delta_{2}^{\omega-1}}\right] \\
& +\sum_{i=1}^{n} \sum_{j=1}^{n}\left|u_{i j}\right| \int_{0}^{\infty} k_{j}(s) F_{j}\left[\frac{\omega-1}{\omega}\left|\xi_{i}(s)\right|^{\omega} \delta_{3}+\frac{1}{\omega}\left|\xi_{j}(s-\tau)\right|^{\omega} \frac{1}{\delta_{3}^{\omega-1}}\right] d s \\
& +\sum_{i=1}^{n} \sum_{j=1}^{n}\left|v_{i j}\right| \int_{0}^{\infty} k_{j}(s) F_{j}\left[\frac{\omega-1}{\omega}\left|\xi_{i}(s)\right|^{\omega} \delta_{3}+\frac{1}{\omega}\left|\xi_{j}(s-\tau)\right|^{\omega} \frac{1}{\delta_{3}^{\omega-1}}\right] d s \\
& \leq-\sum_{i=1}^{n}\left[d_{i}+\left|\phi_{i}(t)\right|+\left|\Psi_{i}(t)\right|\right]\left|\xi_{i}(t)\right|^{\omega}+\sum_{i=1}^{n} \sum_{j=1}^{n}\left|c_{i j}\right| F_{j}\left[\frac{\omega-1}{\omega}\left|\xi_{i}(t)\right|^{\omega} \delta_{1}\right] \\
& +\sum_{i=1}^{n} \sum_{j=1}^{n}\left|c_{i j}\right| F_{j}\left[\frac{1}{\omega}\left|\xi_{j}(t)\right|^{\omega} \frac{1}{\delta_{1}^{\omega-1}}\right]+\sum_{i=1}^{n} \sum_{j=1}^{n}\left|a_{i j}\right| F_{j}\left[\frac{\omega-1}{\omega}\left|\xi_{i}(t)\right|^{\omega} \delta_{2}\right] \\
& \left.+\sum_{i=1}^{n} \sum_{j=1}^{n}\left|a_{i j}\right| F_{j}\right]\left[\frac{1}{\omega}\left|\xi_{j}(t-\tau)\right|^{\omega} \frac{1}{\delta_{2}^{\omega-1}}\right]+\sum_{i=1}^{n} \sum_{j=1}^{n}\left|u_{i j}\right| \int_{0}^{\infty} k_{j}(s) F_{j}\left[\frac{\omega-1}{\omega}\left|\xi_{i}(s)\right|^{\omega} \delta_{3}\right] d s \\
& +\sum_{i=1}^{n} \sum_{j=1}^{n}\left|u_{i j}\right| \int_{0}^{\infty} k_{j}(s) F_{j}\left[\frac{1}{\omega}\left|\xi_{j}(s-\tau)\right|^{\omega} \frac{1}{\delta_{3}^{\omega-1}}\right] d s \\
& +\sum_{i=1}^{n} \sum_{j=1}^{n}\left|v_{i j}\right| \int_{0}^{\infty} k_{j}(s) F_{j}\left[\frac{\omega-1}{\omega}\left|\xi_{i}(s)\right|^{\omega} \delta_{3}\right] d s \\
& +\sum_{i=1}^{n} \sum_{j=1}^{n}\left|v_{i j}\right| \int_{0}^{\infty} k_{j}(s) F_{j}\left[\frac{1}{\omega}\left|\xi_{j}(s-\tau)\right|^{\omega} \frac{1}{\delta_{3}^{\omega-1}}\right] d s \\
& \leq-\sum_{i=1}^{n}\left[d_{i}+\left|\phi_{i}(t)\right|+\left|\Psi_{i}(t)\right|\right]\left|\xi_{i}(t)\right|^{\omega}+\sum_{i=1}^{n} \sum_{j=1}^{n}\left|c_{i j}\right| F_{j}\left[\frac{\omega-1}{\omega}\left|\xi_{i}(t)\right|^{\omega} \delta_{1}\right] \\
& +\sum_{i=1}^{n} \sum_{j=1}^{n}\left|c_{j i}\right| F_{i}\left[\frac{1}{\omega}\left|\xi_{i}(t)\right|^{\omega} \frac{1}{\delta_{1}^{\omega-1}}\right] \\
& \left.+\sum_{i=1}^{n} \sum_{j=1}^{n}\left|a_{i j}\right| F_{j}\left[\frac{\omega-1}{\omega}\left|\xi_{i}(t)\right|^{\omega} \delta_{2}\right]+\sum_{i=1}^{n} \sum_{j=1}^{n}\left|a_{j i}\right| F_{i}\right]\left[\frac{1}{\omega}\left|\xi_{i}(t-\tau)\right|^{\omega} \frac{1}{\delta_{2}^{\omega-1}}\right] \\
& +\sum_{i=1}^{n} \sum_{j=1}^{n}\left|u_{i j}\right| \int_{0}^{\infty} k_{j}(s) F_{j}\left[\frac{\omega-1}{\omega}\left|\xi_{i}(s)\right|^{\omega} \delta_{3}\right] d s
\end{aligned}
$$




$$
\begin{aligned}
& +\sum_{i=1}^{n} \sum_{j=1}^{n}\left|u_{j i}\right| \int_{0}^{\infty} k_{j}(s) F_{i}\left[\frac{1}{\omega}\left|\xi_{i}(s-\tau)\right|^{\omega} \frac{1}{\delta_{3}^{\omega-1}}\right] d s \\
& +\sum_{i=1}^{n} \sum_{j=1}^{n}\left|v_{i j}\right| \int_{0}^{\infty} k_{j}(s) F_{j}\left[\frac{\omega-1}{\omega}\left|\xi_{i}(s)\right|^{\omega} \delta_{3}\right] d s \\
& +\sum_{i=1}^{n} \sum_{j=1}^{n}\left|v_{j i}\right| \int_{0}^{\infty} k_{j}(s) F_{i}\left[\frac{1}{\omega}\left|\xi_{i}(s-\tau)\right|^{\omega} \frac{1}{\delta_{3}^{\omega-1}}\right] d s \\
& \leq \sum_{i=1}^{n}\left[-\omega\left(d_{i}+\left|\phi_{i}(t)\right|+\left|\Psi_{i}(t)\right|\right)+\sum_{j=1}^{n}\left|c_{i j}\right| F_{j} \delta_{1}(\omega-1)\right. \\
& \left.+\sum_{j=1}^{n}\left|c_{j i}\right| F_{i} \frac{1}{\delta_{1}^{\omega-1}}+\sum_{j=1}^{n}\left|a_{i j}\right| F_{j} \delta_{2}(\omega-1)\right] \frac{\left|\xi_{i}(t)\right|^{\omega}}{\omega}+\sum_{i=1}^{n}\left[\sum_{j=1}^{n}\left|a_{j i}\right| F_{i} \frac{1}{\delta_{2}^{\omega-1}}\right] \frac{\left|\xi_{i}(t-\tau)\right|^{\omega}}{\omega} \\
& +\sum_{i=1}^{n} \sum_{j=1}^{n} \int_{0}^{\infty} k_{j}(s)\left[\left|u_{i j}\right| F_{j}(\omega-1) \delta_{3}+\left|v_{i j}\right| F_{j}(\omega-1) \delta_{3}\right] \frac{\left|\xi_{i}(s)\right|^{\omega}}{\omega} d s \\
& +\sum_{i=1}^{n} \sum_{j=1}^{n} \int_{0}^{\infty} k_{j}(s)\left[\left|u_{j i}\right| F_{i}\left(\frac{1}{\delta_{3}^{\omega-1}}\right)+\left|v_{j i}\right| F_{i}\left(\frac{1}{\delta_{3}^{\omega-1}}\right)\right] \frac{\left|\xi_{i}(s-\tau)\right|^{\omega}}{\omega} d s \\
& \leq-\sum_{i=1}^{n}\left[\omega\left(d_{i}+\left|\phi_{i}(t)\right|+\left|\Psi_{i}(t)\right|\right)-\sum_{j=1}^{n}\left|c_{i j}\right| F_{j} \delta_{1}(\omega-1)\right. \\
& \left.-\sum_{j=1}^{n}\left|c_{j i}\right| F_{i} \frac{1}{\delta_{1}^{\omega-1}}-\sum_{j=1}^{n}\left|a_{i j}\right| F_{j} \delta_{2}(\omega-1)\right] \frac{\left|\xi_{i}(t)\right|^{\omega}}{\omega}+\sum_{i=1}^{n}\left[\sum_{j=1}^{n}\left|a_{j i}\right| F_{i} \frac{1}{\delta_{2}^{\omega-1}}\right] \frac{\left|\xi_{i}(t-\tau)\right|^{\omega}}{\omega} \\
& +\sum_{i=1}^{n} \sum_{j=1}^{n} \int_{0}^{\infty} k_{j}(s)\left[\left|u_{i j}\right| F_{j}(\omega-1) \delta_{3}+\left|v_{i j}\right| F_{j}(\omega-1) \delta_{3}\right] \frac{\left|\xi_{i}(s)\right|^{\omega}}{\omega} d s \\
& +\sum_{i=1}^{n} \sum_{j=1}^{n} \int_{0}^{\infty} k_{j}(s)\left[\left|u_{j i}\right| F_{i}\left(\frac{1}{\delta_{3}^{\omega-1}}\right)+\left|v_{j i}\right| F_{i}\left(\frac{1}{\delta_{3}^{\omega-1}}\right)\right] \frac{\left|\xi_{i}(s-\tau)\right|^{\omega}}{\omega} d s \\
& \leq-\theta_{1}(t) V(t, \xi(t))+\theta_{2} \max _{t-\tau \leq s \leq t} V(s, \xi(s)) \text {. }
\end{aligned}
$$

where

$$
\begin{aligned}
\theta_{1}(t) & =\min _{1 \leq i \leq n}\left[\omega\left(d_{i}+\left|\phi_{i}(t)\right|+\left|\Psi_{i}(t)\right|\right)-\sum_{j=1}^{n}\left|c_{i j}\right| F_{j} \delta_{1}(\omega-1)-\sum_{j=1}^{n}\left|c_{j i}\right| F_{i} \frac{1}{\delta_{1}^{\omega-1}}\right. \\
& \left.-\sum_{j=1}^{n}\left|a_{i j}\right| F_{j}(\omega-1) \delta_{2}+\left|u_{i j}\right| F_{j}(\omega-1) \delta_{3}+\left|v_{i j}\right| F_{j}(\omega-1) \delta_{3}\right] \frac{\left|\xi_{i}(t)\right|^{\omega}}{\omega} \\
\theta_{2}(t) & =\max _{1 \leq i \leq n}\left[\sum_{j=1}^{n}\left|a_{j i}\right| F_{i} \frac{1}{\delta_{2}^{\omega-1}}+\sum_{j=1}^{n}\left|u_{j i}\right| F_{i} \frac{1}{\delta_{3}^{\omega-1}}+\sum_{j=1}^{n}\left|v_{j i}\right| F_{i} \frac{1}{\delta_{3}^{\omega-1}} \frac{\left|\xi_{i}(t-\tau)\right|^{\omega}}{\omega}\right.
\end{aligned}
$$

From this estimate, for any solution (13), which satisfies the Razumichin condition

$$
V(s, \xi(s)) \leq V(t, \xi(t)), t-\tau \leq s \leq t
$$

we have

$$
{ }^{C} D^{\alpha} V(t, \xi(t)) \leq-\left(\eta_{1}-\eta_{2}\right) V(t, \xi(t)), t \geq 0 .
$$

According to Lemma 5, the claim of Theorem 4.2 follows.

$$
V(t, \xi(t)) \leq V(0, \xi(0)) E_{\alpha}\left(-\gamma t^{\alpha}\right), t \geq 0
$$


By Lemma 4 and inequality (25), this proves the system (10) can be achieved globally Mittag-Leffler stabilization under the designed control law. Hence complete the proof.

Theorem 4.4. Assume that Assumption (1) and Lemma (4) hold, then the system (10) is Globally mittag-leffler stable, provided the following conditions holds:

$$
\begin{aligned}
\eta_{1}(t) & >\eta_{2}(t) \\
\eta_{1}(t) & =\min _{1 \leq i \leq}\left[\left(d_{i}+\phi_{i}+\Psi_{i}\right)-\sum_{j=1}^{n} c_{i j} F_{j} \rho^{-1}-\sum_{j=1}^{n} c_{j i} F_{i} \rho-\sum_{j=1}^{n} a_{i j} F_{j} \rho^{-1}\right. \\
& \left.+\sum_{j=1}^{n}\left|u_{i j}\right| F_{j} \rho^{-1}+\sum_{j=1}^{n}\left|v_{i j}\right| F_{j} \rho^{-1}\right]>0 \\
\eta_{2}(t) & =\max _{1 \leq i \leq n}\left[\sum_{j=1}^{n} a_{j i} F_{i} \rho+\sum_{j=1}^{n}\left|u_{j i}\right| F_{i} \rho+\sum_{j=1}^{n}\left|v_{j i}\right| F_{i} \rho\right]>0
\end{aligned}
$$

Proof:Let us consider the Lyapunov function is

$$
\begin{gathered}
V(t)=\sum_{i=1}^{n} \frac{1}{2} \xi_{i}^{2}(t)+\sum_{i=1}^{n} \frac{1}{2 q_{i}}\left(\phi_{i}(t)-\phi_{i}\right)^{2}+\sum_{i=1}^{n} \frac{1}{2 r_{i}}\left(\Psi_{i}(t)-\Psi_{i}\right)^{2} \\
{ }^{C} D^{\alpha} \phi_{i}(t)=q_{i} \xi_{i}^{2}(t), \quad{ }^{C} D^{\alpha} \Psi_{i}(t)=r_{i} \xi_{i}^{2}(t)
\end{gathered}
$$

According to the Caputo fractional-order derivative definition, we have

$$
\begin{aligned}
{ }^{C} D^{\alpha} V(t, \xi(t)) \leq & \sum_{i=1}^{n} \xi_{i}(t)^{C} D^{\alpha} \xi_{i}(t)+\sum_{i=1}^{n} \frac{1}{q_{i}}\left(\phi_{i}(t)-\phi_{i}\right)^{C} D^{\alpha} \phi_{i}(t)+\sum_{i=1}^{n} \frac{1}{r_{i}}\left(\Psi_{i}(t)-\Psi_{i}\right)^{C} D^{\alpha} \psi_{i}(t) \\
& \leq \sum_{i=1}^{n} \xi_{i}(t)\left[-d_{i} \xi_{i}(t)+\sum_{j=1}^{n} c_{i j}\left[f_{j}\left(\vartheta_{j}(t)\right)-f_{j}\left(\zeta_{j}(t)\right)\right]\right. \\
& +\sum_{j=1}^{n} a_{i j}\left[f_{j}\left(\vartheta_{j}(t-\tau)\right)-f_{j}\left(\zeta_{j}(t-\tau)\right)\right] \\
& +\bigwedge_{j=1}^{n}\left|u_{i j}\right| \int_{0}^{\infty} k_{j}(s)\left[f_{j}\left(\vartheta_{j}(t-\tau)\right)-f_{j}\left(\zeta_{j}(t-\tau)\right)\right] d s \\
& +\bigvee_{j=1}^{n}\left|v_{i j}\right| \int_{0}^{\infty} k_{j}(s)\left[f_{j}\left(\vartheta_{j}(t-\tau)\right)-f_{j}\left(\zeta_{j}(t-\tau)\right)\right] d s-\phi_{i}(t) \xi_{i}(t) \\
& \left.-\Psi_{i}(t) \xi_{i}(t)\right]+\sum_{i=1}^{n} \frac{1}{q_{i}}\left(\phi_{i}(t)-\phi_{i}\right) q_{i} \xi_{i}^{2}(t)+\sum_{i=1}^{n} \frac{1}{r_{i}}\left(\Psi_{i}(t)-\Psi_{i}\right) r_{i} \xi_{i}^{2}(t) \\
& =-\sum_{i=1}^{n} d_{i} \xi_{i}^{2}(t)+\sum_{i=1}^{n} \sum_{j=1}^{n} c_{i j} F_{j}\left|\xi_{i}(t)\left\|\xi_{j}(t)\left|+\sum_{i=1}^{n} \sum_{j=1}^{n} a_{i j} F_{j}\right| \xi_{i}(t)\right\| \xi_{j}(t-\tau)\right| \\
& +\sum_{i=1}^{n} \sum_{j=1}^{n}\left|u_{i j}\right| \int_{0}^{\infty} k_{j}(s) F_{j}\left|\xi_{i}(s)\right|\left|\xi_{j}(s-\tau)\right| d s \\
& +\sum_{i=1}^{n} \sum_{j=1}^{n}\left|v_{i j}\right| \int_{0}^{\infty} k_{j}(s) F_{j}\left|\xi_{i}(s)\right|\left|\xi_{j}(s-\tau)\right| d s-\sum_{i=1}^{n} \phi_{i}(t) \xi_{i}^{2}(t)-\sum_{i=1}^{n} \Psi_{i}(t) \xi_{i}^{2}(t) \\
& +\sum_{i=1}^{n}\left(\phi_{i}(t)-\phi_{i}\right) \xi_{i}^{2}(t)+\sum_{i=1}^{n}\left(\Psi_{i}(t)-\Psi_{i}\right) \xi_{i}^{2}(t) \\
&
\end{aligned}
$$




$$
\begin{aligned}
& \leq-\sum_{i=1}^{n} d_{i} \xi_{i}^{2}(t)+\sum_{i=1}^{n} \sum_{j=1}^{n} c_{i j} F_{j}\left[\frac{\rho^{-1}}{2} \xi_{i}^{2}(t)+\frac{\rho}{2} \xi_{j}^{2}(t)\right] \\
& +\sum_{i=1}^{n} \sum_{j=1}^{n} a_{i j} F_{j}\left[\frac{\rho^{-1}}{2} \xi_{i}^{2}(t)+\frac{\rho}{2} \xi_{j}^{2}(t-\tau)\right] \\
& +\sum_{i=1}^{n} \sum_{j=1}^{n}\left|u_{i j}\right| \int_{0}^{\infty} k_{j}(s) F_{j}\left[\frac{\rho^{-1}}{2} \xi_{i}^{2}(s)+\frac{\rho}{2} \xi_{j}^{2}(s-\tau)\right] d s \\
& +\sum_{i=1}^{n} \sum_{j=1}^{n}\left|v_{i j}\right| \int_{0}^{\infty} k_{j}(s) F_{j}\left[\frac{\rho^{-1}}{2} \xi_{i}^{2}(s)+\frac{\rho}{2} \xi_{j}^{2}(s-\tau)\right] d s-\sum_{i=1}^{n} \phi_{i}(t) \xi_{i}^{2}(t)-\sum_{i=1}^{n} \Psi_{i}(t) \xi_{i}^{2}(t) \\
& +\sum_{i=1}^{n} \phi_{i}(t) \xi_{i}^{2}(t)-\sum_{i=1}^{n} \phi_{i} \xi_{i}^{2}(t)+\sum_{i=1}^{n} \Psi_{i}(t) \xi_{i}^{2}(t)-\sum_{i=1}^{n} \Psi_{i} \xi_{i}^{2}(t) \\
& \leq-\sum_{i=1}^{n} d_{i} \xi_{i}^{2}(t)+\sum_{i=1}^{n} \sum_{j=1}^{n} c_{i j} F_{j}\left[\frac{\rho^{-1}}{2} \xi_{i}^{2}(t)\right]+\sum_{i=1}^{n} \sum_{j=1}^{n} c_{i j} F_{j}\left[\frac{\rho}{2} \xi_{j}^{2}(t)\right] \\
& +\sum_{i=1}^{n} \sum_{j=1}^{n} a_{i j} F_{j}\left[\frac{\rho^{-1}}{2} \xi_{i}^{2}(t)\right]+\sum_{i=1}^{n} \sum_{j=1}^{n} a_{i j} F_{j}\left[\frac{\rho}{2} \xi_{j}^{2}(t-\tau)\right] \\
& +\sum_{i=1}^{n} \sum_{j=1}^{n}\left|u_{i j}\right| \int_{0}^{\infty} k_{j}(s) F_{j}\left[\frac{\rho^{-1}}{2} \xi_{i}^{2}(s)\right] d s+\sum_{i=1}^{n} \sum_{j=1}^{n}\left|u_{i j}\right| \int_{0}^{\infty} k_{j}(s) F_{j}\left[\frac{\rho}{2} \xi_{j}^{2}(s-\tau)\right] d s \\
& +\sum_{i=1}^{n} \sum_{j=1}^{n}\left|v_{i j}\right| \int_{0}^{\infty} k_{j}(s) F_{j}\left[\frac{\rho^{-1}}{2} \xi_{i}^{2}(s)\right] d s+\sum_{i=1}^{n} \sum_{j=1}^{n}\left|v_{i j}\right| \int_{0}^{\infty} k_{j}(s) F_{j}\left[\frac{\rho}{2} \xi_{j}^{2}(s-\tau)\right] d s \\
& -\sum_{i=1}^{n} \phi_{i} \xi_{i}^{2}(t)-\sum_{i=1}^{n} \Psi_{i} \xi_{i}^{2}(t) \\
& \leq-\sum_{i=1}^{n} d_{i} \xi_{i}^{2}(t)+\sum_{i=1}^{n} \sum_{j=1}^{n} c_{i j} F_{j}\left[\frac{\rho^{-1}}{2} \xi_{i}^{2}(t)\right]+\sum_{i=1}^{n} \sum_{j=1}^{n} c_{j i} F_{i}\left[\frac{\rho}{2} \xi_{i}^{2}(t)\right] \\
& +\sum_{i=1}^{n} \sum_{j=1}^{n} a_{i j} F_{j}\left[\frac{\rho^{-1}}{2} \xi_{i}^{2}(t)\right]+\sum_{i=1}^{n} \sum_{j=1}^{n} a_{j i} F_{i}\left[\frac{\rho}{2} \xi_{i}^{2}(t-\tau)\right] \\
& +\sum_{i=1}^{n} \sum_{j=1}^{n}\left|u_{i j}\right| \int_{0}^{\infty} k_{j}(s) F_{j}\left[\frac{\rho^{-1}}{2} \xi_{i}^{2}(s)\right] d s+\sum_{i=1}^{n} \sum_{j=1}^{n}\left|u_{j i}\right| \int_{0}^{\infty} k_{j}(s) F_{i}\left[\frac{\rho}{2} \xi_{i}^{2}(s-\tau)\right] d s \\
& +\sum_{i=1}^{n} \sum_{j=1}^{n}\left|v_{i j}\right| \int_{0}^{\infty} k_{j}(s) F_{j}\left[\frac{\rho^{-1}}{2} \xi_{i}^{2}(s)\right] d s+\sum_{i=1}^{n} \sum_{j=1}^{n}\left|v_{j i}\right| \int_{0}^{\infty} k_{j}(s) F_{i}\left[\frac{\rho}{2} \xi_{i}^{2}(s-\tau)\right] d s \\
& -\sum_{i=1}^{n} \phi_{i} \xi_{i}^{2}(t)-\sum_{i=1}^{n} \Psi_{i} \xi_{i}^{2}(t) \\
& \leq \sum_{i=1}^{n}\left[-\left(d_{i}+\phi_{i}+\Psi_{i}\right)+\sum_{j=1}^{n} c_{i j} F_{j} \frac{\rho^{-1}}{2}+\sum_{j=1}^{n} c_{j i} F_{i} \frac{\rho}{2}+\sum_{j=1}^{n} a_{i j} F_{j}\left[\frac{\rho^{-1}}{2}\right]\right] \xi_{i}^{2}(t) \\
& +\sum_{i=1}^{n}\left[\sum_{j=1}^{n} a_{j i} F_{i}\left[\frac{\rho}{2}\right]\right] \xi_{i}^{2}(t-\tau)+\sum_{i=1}^{n}\left[\sum_{j=1}^{n}\left|u_{i j}\right| \int_{0}^{\infty} k_{j}(s) F_{j}\left[\frac{\rho^{-1}}{2}\right] d s\right. \\
& \left.+\sum_{j=1}^{n}\left|v_{i j}\right| \int_{0}^{\infty} k_{j}(s) F_{j}\left[\frac{\rho^{-1}}{2}\right]\right] \xi_{i}^{2}(s) d s+\sum_{i=1}^{n}\left[\sum_{j=1}^{n}\left|u_{j i}\right| \int_{0}^{\infty} k_{j}(s) F_{i}\left[\frac{\rho}{2}\right] d s\right.
\end{aligned}
$$




$$
\begin{aligned}
& \left.+\sum_{j=1}^{n}\left|v_{j i}\right| \int_{0}^{\infty} k_{j}(s) F_{i}\left[\frac{\rho}{2}\right]\right] \xi_{i}^{2}(s-\tau) d s \\
& \leq-\sum_{i=1}^{n}\left[\left(d_{i}+\phi_{i}+\Psi_{i}\right)-\sum_{j=1}^{n} c_{i j} F_{j} \frac{\rho^{-1}}{2}-\sum_{j=1}^{n} c_{j i} F_{i} \frac{\rho}{2}-\sum_{j=1}^{n} a_{i j} F_{j}\left[\frac{\rho^{-1}}{2}\right]\right] \xi_{i}^{2}(t) \\
& +\sum_{i=1}^{n}\left[\sum_{j=1}^{n} a_{j i} F_{i}\left[\frac{\rho}{2}\right]\right] \xi_{i}^{2}(t-\tau)+\sum_{i=1}^{n}\left[\sum_{j=1}^{n}\left|u_{i j}\right| i n t_{0}^{\infty} k_{j}(s) F_{j}\left[\frac{\rho^{-1}}{2}\right] d s\right. \\
& \left.+\sum_{j=1}^{n}\left|v_{i j}\right| \int_{0}^{\infty} k_{j}(s) F_{j}\left[\frac{\rho^{-1}}{2}\right]\right] \xi_{i}^{2}(s) d s+\sum_{i=1}^{n}\left[\sum_{j=1}^{n}\left|u_{j i}\right| \int_{0}^{\infty} k_{j}(s) F_{i}\left[\frac{\rho}{2}\right] d s\right. \\
& \left.+\sum_{j=1}^{n}\left|v_{j i}\right| \int_{0}^{\infty} k_{j}(s) F_{i}\left[\frac{\rho}{2}\right]\right] \xi_{i}^{2}(s-\tau)(s) d s \\
& \leq-\eta_{1} V(t, \xi(t))+\eta_{2} \max _{t-\tau \leq s \leq t} V(s, \xi(s))
\end{aligned}
$$

where,

$$
\begin{aligned}
\eta_{1}(t) & =\min _{1 \leq i \leq n}\left[\left(d_{i}+\phi_{i}+\Psi_{i}\right)-\sum_{j=1}^{n} c_{i j} F_{j} \rho^{-1}-\sum_{j=1}^{n} c_{j i} F_{i} \rho-\sum_{j=1}^{n} a_{i j} F_{j} \rho^{-1}\right. \\
& \left.+\sum_{j=1}^{n}\left|u_{i j}\right| F_{j} \rho^{-1}+\sum_{j=1}^{n}\left|v_{i j}\right| F_{j} \rho^{-1}\right]>0, \\
\eta_{2}(t) & =\max _{1 \leq i \leq n}\left[\sum_{j=1}^{n} a_{j i} F_{i} \rho+\sum_{j=1}^{n}\left|u_{j i}\right| F_{i} \rho+\sum_{j=1}^{n}\left|v_{j i}\right| F_{i} \rho\right]>0 .
\end{aligned}
$$

From this estimate, for any solution (13), which satisfies the Razumichin condition

$$
V(s, \xi(s)) \leq V(t, \xi(t)), t-\tau \leq s \leq t
$$

we have

$$
{ }^{C} D^{\alpha} V(t, \xi(t)) \leq-\left(\eta_{1}-\eta_{2}\right) V(t, \xi(t)), t \geq 0
$$

According to Lemma 5, the claim of Theorem 4.3 follows.

$$
V(t, \xi(t)) \leq V(0, \xi(0)) E_{\alpha}\left(-\gamma t^{\alpha}\right), t \geq 0
$$

By Lemma 4 and inequality (31), this proves system (10) can be achieved globally Mittag-Leffler stabilization under the designed control law.

This completes the proof.

Theorem 4.5. Assume that Assumption (1) and Lemma (4) hold, then the system (10) is Globally mittag-leffler stable, provided the following conditions holds:

$$
\begin{aligned}
\theta_{1}(t) & >\theta_{2}(t) \\
\theta_{1}(t) & =\min _{1 \leq i \leq n}\left[\omega\left(d_{i}+\left|\phi_{i}(t)\right|+\left|\Psi_{i}(t)\right|\right)-\sum_{j=1}^{n}\left|c_{i j}\right| F_{j} \delta_{1}(\omega-1)-\sum_{j=1}^{n}\left|c_{j i}\right| F_{i} \frac{1}{\delta_{1}^{\omega-1}}\right. \\
& \left.-\sum_{j=1}^{n}\left|a_{i j}\right| F_{j}(\omega-1) \delta_{2}+\left|u_{i j}\right| F_{j}(\omega-1) \delta_{3}+\left|v_{i j}\right| F_{j}(\omega-1) \delta_{3}\right] \frac{\left|\xi_{i}(t)\right|^{\omega}}{\omega} \\
& +\min _{1 \leq i \leq n}\left[\rho\left(d_{i}+\left|\phi_{i}(t)\right|+\left|\Psi_{i}(t)\right|\right)-\sum_{j=1}^{n}\left|c_{i j}\right| F_{j} \delta_{1}(\rho-1)-\sum_{j=1}^{n}\left|c_{j i}\right| F_{i} \frac{1}{\delta_{1}^{\rho-1}}\right.
\end{aligned}
$$




$$
\begin{aligned}
& \left.-\sum_{j=1}^{n}\left|a_{i j}\right| F_{j}(\rho-1) \delta_{2}+\left|u_{i j}\right| F_{j}(\rho-1) \delta_{3}+\left|v_{i j}\right| F_{j}(\rho-1) \delta_{3}\right] \frac{\left|\xi_{i}(t)\right|^{\rho}}{\rho}>0, \\
\theta_{2}(t) & =\max _{1 \leq i \leq n}\left[\sum_{j=1}^{n}\left|a_{j i}\right| F_{i} \frac{1}{\delta_{2}^{\omega-1}}+\sum_{j=1}^{n}\left|u_{j i}\right| F_{i} \frac{1}{\delta_{3}^{\omega-1}}+\sum_{j=1}^{n}\left|v_{j i}\right| F_{i} \frac{1}{\delta_{3}^{\omega-1}}\right] \frac{\left|\xi_{i}(t-\tau)\right|^{\omega}}{\omega} \\
& +\max _{1 \leq i \leq n}\left[\sum_{j=1}^{n}\left|a_{j i}\right| F_{i} \frac{1}{\delta_{2}^{\rho-1}}+\sum_{j=1}^{n}\left|u_{j i}\right| F_{i} \frac{1}{\delta_{3}^{\rho-1}}+\sum_{j=1}^{n}\left|v_{j i}\right| F_{i} \frac{1}{\delta_{3}^{\rho-1}}\right] \frac{\left|\xi_{i}(t-\tau)\right|^{\rho}}{\rho}>0 .
\end{aligned}
$$

Proof: we define a Lyapunov function

$$
V(t, \xi(t))=\sum_{i=1}^{n} \frac{1}{\omega}\left|\xi_{i}(t)\right|^{\omega}+\sum_{i=1}^{n} \frac{1}{\rho}\left|\xi_{i}(t)\right|^{\rho}
$$

According to the Caputo fractional-order derivative definition, we have

$$
\begin{aligned}
{ }^{C} D^{\alpha} V(t, \xi(t)) \leq & \sum_{i=1}^{n}\left|\xi_{i}(t)\right|^{\omega-1}\left[-d_{i}\left|\xi_{i}(t)\right|+\sum_{j=1}^{n}\left|c_{i j}\right|\left[f_{j}\left(\vartheta_{j}(t)\right)-f_{j}\left(\zeta_{j}(t)\right)\right]\right. \\
& +\sum_{j=1}^{n}\left|a_{i j}\right|\left[f_{j}\left(\vartheta_{j}(t-\tau)\right)-f_{j}\left(\zeta_{j}(t-\tau)\right)\right] \\
& +\bigwedge_{j=1}^{n}\left|u_{i j}\right| \int_{0}^{\infty} k_{j}(s)\left[f_{j}\left(\vartheta_{j}(s-\tau)\right)-f_{j}\left(\zeta_{j}(t-\tau)\right)\right] d s \\
& \left.+\bigvee_{j=1}^{n}\left|v_{i j}\right| \int_{0}^{\infty} k_{j}(s)\left[f_{j}\left(\vartheta_{j}(s-\tau)\right)-f_{j}\left(\zeta_{j}(t-\tau)\right)\right] d s+\left|\phi_{i}(t)\right|\left|\xi_{i}(t)\right|+\left|\Psi_{i}(t)\right|\left|\xi_{i}(t)\right|\right] \\
& +\sum_{i=1}^{n}\left|\xi_{i}(t)\right|^{\rho-1}\left[-d_{i}\left|\xi_{i}(t)\right|+\sum_{j=1}^{n}\left|c_{i j}\right|\left[f_{j}\left(\vartheta_{j}(t)\right)-f_{j}\left(\zeta_{j}(t)\right)\right]\right. \\
& +\sum_{j=1}^{n}\left|a_{i j}\right|\left[f_{j}\left(\vartheta_{j}(t-\tau)\right)-f_{j}\left(\zeta_{j}(t-\tau)\right)\right] \\
& +\bigwedge_{j=1}^{n}\left|u_{i j}\right| \int_{0}^{\infty} k_{j}(s)\left[f_{j}\left(\vartheta_{j}(s-\tau)\right)-f_{j}\left(\zeta_{j}(t-\tau)\right)\right] d s \\
& \left.+\bigvee_{j=1}^{n}\left|v_{i j}\right| \int_{0}^{\infty} k_{j}(s)\left[f_{j}\left(\vartheta_{j}(s-\tau)\right)-f_{j}\left(\zeta_{j}(t-\tau)\right)\right] d s+\left|\phi_{i}(t)\right|\left|\xi_{i}(t)\right|+\left|\Psi_{i}(t)\right|\left|\xi_{i}(t)\right|\right] \\
& \leq \sum_{i=1}^{n}\left|\xi_{i}(t)\right|^{\omega-1}\left[-d_{i}\left|\xi_{i}(t)\right|+\sum_{j=1}^{n}\left|c_{i j}\right| F_{j}\left|\xi_{j}(t)\right|+\sum_{j=1}^{n}\left|a_{i j}\right| F_{j}\left|\xi_{j}(t-\tau)\right|\right. \\
& +\sum_{j=1}^{n}\left|u_{i j}\right| \int_{0}^{\infty} k_{j}(s) F_{j}\left|\xi_{j}(s-\tau)\right| d s+\sum_{j=1}^{n}\left|v_{i j}\right| \int_{0}^{\infty} k_{j}(s) F_{j}\left|\xi_{j}(s-\tau)\right| d s \\
& \left.+\left|\phi_{i}(t)\right|\left|\xi_{i}(t)\right|+\left|\Psi_{i}(t)\right|\left|\xi_{i}(t)\right|\right] \\
& +\sum_{i=1}^{n}\left|\xi_{i}(t)\right|^{\rho-1}\left[-d_{i}\left|\xi_{i}(t)\right|+\sum_{j=1}^{n}\left|c_{i j}\right| F_{j}\left|\xi_{j}(t)\right|+\sum_{j=1}^{n}\left|a_{i j}\right| F_{j}\left|\xi_{j}(t-\tau)\right|\right. \\
& \left.\left|\phi_{i}(t)\right|\left|\xi_{i}(t)\right|+\left|\Psi_{i}(t)\right|\left|\xi_{i}(t)\right|\right] \\
& k_{j}(s) F_{j}\left|\xi_{j}(s-\tau)\right| d s+\sum_{j=1}^{\infty}\left|v_{i j}\right| \int_{0}^{\infty} k_{j}(s) F_{j}\left|\xi_{j}(s-\tau)\right| d s \\
& \\
& \\
&
\end{aligned}
$$




$$
\begin{aligned}
& \leq-\sum_{i=1}^{n}\left(d_{i}+\left|\phi_{i}(t)\right|+\left|\Psi_{i}(t)\right|\right)\left|\xi_{i}(t)\right|^{\omega}+\sum_{i=1}^{n} \sum_{j=1}^{n}\left|c_{i j}\right| F_{j}\left|\xi_{i}(t)\right|^{\omega-1}\left|\xi_{j}(t)\right| \\
& +\sum_{i=1}^{n} \sum_{j=1}^{n}\left|a_{i j}\right| F_{j}\left|\xi_{i}(t)\right|^{\omega-1}\left|\xi_{j}(t-\tau)\right|+\sum_{i=1}^{n} \sum_{j=1}^{n}\left|u_{i j}\right| \int_{0}^{\infty} k_{j}(s) F_{j}\left|\xi_{i}(s)\right|^{\omega-1}\left|\xi_{j}(s-\tau)\right| d s \\
& +\sum_{i=1}^{n} \sum_{j=1}^{n}\left|v_{i j}\right| \int_{0}^{\infty} k_{j}(s) F_{j}\left|\xi_{i}(s)\right|^{\omega-1}\left|\xi_{j}(s-\tau)\right| d s \\
& -\sum_{i=1}^{n}\left(d_{i}+\left|\phi_{i}(t)\right|+\left|\Psi_{i}(t)\right|\right)\left|\xi_{i}(t)\right|^{\rho}+\sum_{i=1}^{n} \sum_{j=1}^{n}\left|c_{i j}\right| F_{j}\left|\xi_{i}(t)\right|^{\rho-1}\left|\xi_{j}(t)\right| \\
& +\sum_{i=1}^{n} \sum_{j=1}^{n}\left|a_{i j}\right| F_{j}\left|\xi_{i}(t)\right|^{\rho-1}\left|\xi_{j}(t-\tau)\right|+\sum_{i=1}^{n} \sum_{j=1}^{n}\left|u_{i j}\right| \int_{0}^{\infty} k_{j}(s) F_{j}\left|\xi_{i}(s)\right|^{\rho-1}\left|\xi_{j}(s-\tau)\right| d s \\
& +\sum_{i=1}^{n} \sum_{j=1}^{n}\left|v_{i j}\right| \int_{0}^{\infty} k_{j}(s) F_{j}\left|\xi_{i}(s)\right|^{\rho-1}\left|\xi_{j}(s-\tau)\right| d s
\end{aligned}
$$

From Lemma 3 we have

$$
\begin{aligned}
& \left|\xi_{i}(t)\right|^{\omega-1}\left|\xi_{j}(t)\right| \leq \frac{\omega-1}{\omega}\left[\left|\xi_{i}(t)\right|^{\omega-1} \delta_{1}^{\frac{\omega-1}{\omega}}\right]^{\frac{\omega}{\omega-1}}+\frac{1}{\omega}\left[\left|\xi_{j}(t)\right| \delta_{1}^{\frac{-\omega-1}{\omega}}\right]^{\omega} \\
& =\frac{\omega-1}{\omega}\left|\xi_{i}(t)\right|^{\omega} \delta_{1}+\frac{1}{\omega}\left|\xi_{j}(t)\right|^{\omega} \frac{1}{\delta_{1}^{\omega-1}} \\
& \left|\xi_{i}(t)\right|^{\omega-1}\left|\xi_{j}(t-\tau)\right| \leq \frac{\omega-1}{\omega}\left[\left|\xi_{i}(t)\right|^{\omega-1} \delta_{2}^{\frac{\omega-1}{\omega}}\right]^{\frac{\omega}{\omega-1}}+\frac{1}{\omega}\left[\left|\xi_{j}(t-\tau)\right| \delta_{2}^{\frac{-\omega-1}{\omega}}\right]^{\omega} \\
& =\frac{\omega-1}{\omega}\left|\xi_{i}(t)\right|^{\omega} \delta_{2}+\frac{1}{\omega}\left|\xi_{j}(t-\tau)\right|^{\omega} \frac{1}{\delta_{2}^{\omega-1}} \\
& \int_{0}^{\infty}\left|\xi_{i}(s)\right|^{\omega-1}\left|\xi_{j}(s-\tau)\right| d s \leq \int_{0}^{\infty} \frac{\omega-1}{\omega}\left[\left|\xi_{i}(s)\right|^{\omega-1} \delta_{3}^{\frac{\omega-1}{\omega}}\right]^{\frac{\omega}{\omega-1}}+\frac{1}{\omega}\left[\left|\xi_{j}(s-\tau)\right| \delta_{3}^{\frac{-\omega-1}{\omega}}\right]^{\omega} \\
& =\int_{0}^{\infty} \frac{\omega-1}{\omega}\left|\xi_{i}(s)\right|^{\omega} \delta_{3}+\frac{1}{\omega}\left|\xi_{j}(s-\tau)\right|^{\omega} \frac{1}{\delta_{3}^{\omega-1}} \\
& \left|\xi_{i}(t)\right|^{\rho-1}\left|\xi_{j}(t)\right| \leq \frac{\rho-1}{\rho}\left[\left|\xi_{i}(t)\right|^{\rho-1} \delta_{1}^{\frac{\rho-1}{\rho}}\right]^{\frac{\rho}{\rho-1}}+\frac{1}{\rho}\left[\left|\xi_{j}(t)\right| \delta_{1}^{\frac{-\rho-1}{\rho}}\right]^{\rho} \\
& =\frac{\rho-1}{\rho}\left|\xi_{i}(t)\right|^{\rho} \delta_{1}+\frac{1}{\rho}\left|\xi_{j}(t)\right|^{\rho} \frac{1}{\delta_{1}^{\rho-1}} \\
& \left|\xi_{i}(t)\right|^{\rho-1}\left|\xi_{j}(t-\tau)\right| \leq \frac{\rho-1}{\rho}\left[\left|\xi_{i}(t)\right|^{\rho-1} \delta_{2}^{\frac{\rho-1}{\rho}}\right]^{\frac{\rho}{\rho-1}}+\frac{1}{\rho}\left[\left|\xi_{j}(t-\tau)\right| \delta_{2}^{\frac{-\rho-1}{\rho}}\right]^{\rho} \\
& =\frac{\rho-1}{\rho}\left|\xi_{i}(t)\right|^{\rho} \delta_{2}+\frac{1}{\rho}\left|\xi_{j}(t-\tau)\right|^{\rho} \frac{1}{\delta_{2}^{\rho-1}} \\
& \int_{0}^{\infty}\left|\xi_{i}(s)\right|^{\rho-1}\left|\xi_{j}(s-\tau)\right| d s \leq \int_{0}^{\infty} \frac{\rho-1}{\rho}\left[\left|\xi_{i}(s)\right|^{\rho-1} \delta_{3}^{\frac{\rho-1}{\rho}}\right]^{\frac{\rho}{\rho-1}}+\frac{1}{\rho}\left[\left|\xi_{j}(s-\tau)\right| \delta_{3}^{\frac{-\rho-1}{\rho}}\right]^{\rho} \\
& =\int_{0}^{\infty} \frac{\rho-1}{\rho}\left|\xi_{i}(s)\right|^{\rho} \delta_{3}+\frac{1}{\rho}\left|\xi_{j}(s-\tau)\right|^{\rho} \frac{1}{\delta_{3}^{\rho-1}}
\end{aligned}
$$


Now substitute the above values in the above equation we get

$$
\begin{aligned}
& { }^{C} D^{\alpha} V(t, \xi(t)) \leq-\sum_{i=1}^{n}\left(d_{i}+\left|\phi_{i}(t)\right|+\left|\Psi_{i}(t)\right|\right)\left|\xi_{i}(t)\right|^{\omega}+\sum_{i=1}^{n} \sum_{j=1}^{n}\left|c_{i j}\right| F_{j}\left[\frac{\omega-1}{\omega}\left|\xi_{i}(t)\right|^{\omega} \delta_{1}\right. \\
& \left.+\frac{1}{\omega}\left|\xi_{j}(t)\right|^{\omega} \frac{1}{\delta_{1}^{\omega-1}}\right]+\sum_{i=1}^{n} \sum_{j=1}^{n}\left|a_{i j}\right| F_{j}\left[\frac{\omega-1}{\omega}\left|\xi_{i}(t)\right|^{\omega} \delta_{2}+\frac{1}{\omega}\left|\xi_{j}(t-\tau)\right|^{\omega} \frac{1}{\delta_{2}^{\omega-1}}\right] \\
& +\sum_{i=1}^{n} \sum_{j=1}^{n}\left|u_{i j}\right| \int_{0}^{\infty} k_{j}(s) F_{j}\left[\frac{\omega-1}{\omega}\left|\xi_{i}(s)\right|^{\omega} \delta_{3}+\frac{1}{\omega}\left|\xi_{j}(s-\tau)\right|^{\omega} \frac{1}{\delta_{3}^{\omega-1}}\right] d s \\
& +\sum_{i=1}^{n} \sum_{j=1}^{n}\left|v_{i j}\right| \int_{0}^{\infty} k_{j}(s) F_{j}\left[\frac{\omega-1}{\omega}\left|\xi_{i}(s)\right|^{\omega} \delta_{3}+\frac{1}{\omega}\left|\xi_{j}(s-\tau)\right|^{\omega} \frac{1}{\delta_{3}^{\omega-1}}\right] d s \\
& -\sum_{i=1}^{n}\left(d_{i}+\left|\phi_{i}(t)\right|+\left|\Psi_{i}(t)\right|\right)\left|\xi_{i}(t)\right|^{\rho}+\sum_{i=1}^{n} \sum_{j=1}^{n}\left|c_{i j}\right| F_{j}\left[\frac{\rho-1}{\rho}\left|\xi_{i}(t)\right|^{\rho} \delta_{1}\right. \\
& \left.+\frac{1}{\rho}\left|\xi_{j}(t)\right|^{\rho} \frac{1}{\delta_{1}^{\rho-1}}\right]+\sum_{i=1}^{n} \sum_{j=1}^{n}\left|a_{i j}\right| F_{j}\left[\frac{\rho-1}{\rho}\left|\xi_{i}(t)\right|^{\rho} \delta_{2}+\frac{1}{\rho}\left|\xi_{j}(t-\tau)\right|^{\rho} \frac{1}{\delta_{2}^{\rho-1}}\right] \\
& +\sum_{i=1}^{n} \sum_{j=1}^{n}\left|u_{i j}\right| \int_{0}^{\infty} k_{j}(s) F_{j}\left[\frac{\rho-1}{\rho}\left|\xi_{i}(s)\right|^{\rho} \delta_{3}+\frac{1}{\rho}\left|\xi_{j}(s-\tau)\right|^{\rho} \frac{1}{\delta_{3}^{\rho-1}}\right] d s \\
& +\sum_{i=1}^{n} \sum_{j=1}^{n}\left|v_{i j}\right| \int_{0}^{\infty} k_{j}(s) F_{j}\left[\frac{\rho-1}{\rho}\left|\xi_{i}(s)\right|^{\rho} \delta_{3}+\frac{1}{\rho}\left|\xi_{j}(s-\tau)\right|^{\rho} \frac{1}{\delta_{3}^{\rho-1}}\right] d s \\
& \leq-\sum_{i=1}^{n}\left[d_{i}+\left|\phi_{i}(t)\right|+\left|\Psi_{i}(t)\right|\right]\left|\xi_{i}(t)\right|^{\omega}+\sum_{i=1}^{n} \sum_{j=1}^{n}\left|c_{i j}\right| F_{j}\left[\frac{\omega-1}{\omega}\left|\xi_{i}(t)\right|^{\omega} \delta_{1}\right] \\
& +\sum_{i=1}^{n} \sum_{j=1}^{n}\left|c_{j i}\right| F_{i}\left[\frac{1}{\omega}\left|\xi_{i}(t)\right|^{\omega} \frac{1}{\delta_{1}^{\omega-1}}\right] \\
& \left.+\sum_{i=1}^{n} \sum_{j=1}^{n}\left|a_{i j}\right| F_{j}\left[\frac{\omega-1}{\omega}\left|\xi_{i}(t)\right|^{\omega} \delta_{2}\right]+\sum_{i=1}^{n} \sum_{j=1}^{n}\left|a_{j i}\right| F_{i}\right]\left[\frac{1}{\omega}\left|\xi_{i}(t-\tau)\right|^{\omega} \frac{1}{\delta_{2}^{\omega-1}}\right] \\
& +\sum_{i=1}^{n} \sum_{j=1}^{n}\left|u_{i j}\right| \int_{0}^{\infty} k_{j}(s) F_{j}\left[\frac{\omega-1}{\omega}\left|\xi_{i}(s)\right|^{\omega} \delta_{3}\right] d s \\
& +\sum_{i=1}^{n} \sum_{j=1}^{n}\left|u_{j i}\right| \int_{0}^{\infty} k_{j}(s) F_{i}\left[\frac{1}{\omega}\left|\xi_{i}(s-\tau)\right|^{\omega} \frac{1}{\delta_{3}^{\omega-1}}\right] d s \\
& +\sum_{i=1}^{n} \sum_{j=1}^{n}\left|v_{i j}\right| \int_{0}^{\infty} k_{j}(s) F_{j}\left[\frac{\omega-1}{\omega}\left|\xi_{i}(s)\right|^{\omega} \delta_{3}\right] d s \\
& +\sum_{i=1}^{n} \sum_{j=1}^{n}\left|v_{j i}\right| \int_{0}^{\infty} k_{j}(s) F_{i}\left[\frac{1}{\omega}\left|\xi_{i}(s-\tau)\right|^{\omega} \frac{1}{\delta_{3}^{\omega-1}}\right] d s \\
& -\sum_{i=1}^{n}\left[d_{i}+\left|\phi_{i}(t)\right|+\left|\Psi_{i}(t)\right|\right]\left|\xi_{i}(t)\right|^{\rho}+\sum_{i=1}^{n} \sum_{j=1}^{n}\left|c_{i j}\right| F_{j}\left[\frac{\rho-1}{\rho}\left|\xi_{i}(t)\right|^{\rho} \delta_{1}\right] \\
& +\sum_{i=1}^{n} \sum_{j=1}^{n}\left|c_{j i}\right| F_{i}\left[\frac{1}{\rho}\left|\xi_{i}(t)\right|^{\rho} \frac{1}{\delta_{1}^{\rho-1}}\right]
\end{aligned}
$$




$$
\begin{aligned}
& \left.+\sum_{i=1}^{n} \sum_{j=1}^{n}\left|a_{i j}\right| F_{j}\left[\frac{\rho-1}{\rho}\left|\xi_{i}(t)\right|^{\rho} \delta_{2}\right]+\sum_{i=1}^{n} \sum_{j=1}^{n}\left|a_{j i}\right| F_{i}\right]\left[\frac{1}{\rho}\left|\xi_{i}(t-\tau)\right|^{\rho} \frac{1}{\delta_{2}^{\rho-1}}\right] \\
& +\sum_{i=1}^{n} \sum_{j=1}^{n}\left|u_{i j}\right| \int_{0}^{\infty} k_{j}(s) F_{j}\left[\frac{\rho-1}{\rho}\left|\xi_{i}(s)\right|^{\rho} \delta_{3}\right] d s \\
& +\sum_{i=1}^{n} \sum_{j=1}^{n}\left|u_{j i}\right| \int_{0}^{\infty} k_{j}(s) F_{i}\left[\frac{1}{\rho}\left|\xi_{i}(s-\tau)\right|^{\rho} \frac{1}{\delta_{3}^{\rho-1}}\right] d s \\
& +\sum_{i=1}^{n} \sum_{j=1}^{n}\left|v_{i j}\right| \int_{0}^{\infty} k_{j}(s) F_{j}\left[\frac{\rho-1}{\rho}\left|\xi_{i}(s)\right|^{\rho} \delta_{3}\right] d s \\
& +\sum_{i=1}^{n} \sum_{j=1}^{n}\left|v_{j i}\right| \int_{0}^{\infty} k_{j}(s) F_{i}\left[\frac{1}{\rho}\left|\xi_{i}(s-\tau)\right|^{\rho} \frac{1}{\delta_{3}^{\rho-1}}\right] d s \\
& \leq \sum_{i=1}^{n}\left[-\omega\left(d_{i}+\left|\phi_{i}(t)\right|+\left|\Psi_{i}(t)\right|\right)+\sum_{j=1}^{n}\left|c_{i j}\right| F_{j} \delta_{1}(\omega-1)\right. \\
& \left.+\sum_{j=1}^{n}\left|c_{j i}\right| F_{i} \frac{1}{\delta_{1}^{\omega-1}}+\sum_{j=1}^{n}\left|a_{i j}\right| F_{j} \delta_{2}(\omega-1)\right] \frac{\left|\xi_{i}(t)\right|^{\omega}}{\omega}+\sum_{i=1}^{n}\left[\sum_{j=1}^{n}\left|a_{j i}\right| F_{i} \frac{1}{\delta_{2}^{\omega-1}}\right] \frac{\left|\xi_{i}(t-\tau)\right|^{\omega}}{\omega} \\
& +\sum_{i=1}^{n} \sum_{j=1}^{n} \int_{0}^{\infty} k_{j}(s)\left[\left|u_{i j}\right| F_{j}(\omega-1) \delta_{3}+\left|v_{i j}\right| F_{j}(\omega-1) \delta_{3}\right] \frac{\left|\xi_{i}(s)\right|^{\omega}}{\omega} d s \\
& +\sum_{i=1}^{n} \sum_{j=1}^{n} \int_{0}^{\infty} k_{j}(s)\left[\left|u_{j i}\right| F_{i}\left(\frac{1}{\delta_{3}^{\omega-1}}\right)+\left|v_{j i}\right| F_{i}\left(\frac{1}{\delta_{3}^{\omega-1}}\right)\right] \frac{\left|\xi_{i}(s-\tau)\right|^{\omega}}{\omega} d s \\
& +\sum_{i=1}^{n}\left[-\rho\left(d_{i}+\left|\phi_{i}(t)\right|+\left|\Psi_{i}(t)\right|\right)+\sum_{j=1}^{n}\left|c_{i j}\right| F_{j} \delta_{1}(\rho-1)\right. \\
& \left.+\sum_{j=1}^{n}\left|c_{j i}\right| F_{i} \frac{1}{\delta_{1}^{\rho-1}}+\sum_{j=1}^{n}\left|a_{i j}\right| F_{j} \delta_{2}(\rho-1)\right] \frac{\left|\xi_{i}(t)\right|^{\rho}}{\rho}+\sum_{i=1}^{n}\left[\sum_{j=1}^{n}\left|a_{j i}\right| F_{i} \frac{1}{\delta_{2}^{\rho-1}}\right] \frac{\left|\xi_{i}(t-\tau)\right|^{\rho}}{\rho} \\
& +\sum_{i=1}^{n} \sum_{j=1}^{n} \int_{0}^{\infty} k_{j}(s)\left[\left|u_{i j}\right| F_{j}(\rho-1) \delta_{3}+\left|v_{i j}\right| F_{j}(\rho-1) \delta_{3}\right] \frac{\left|\xi_{i}(s)\right|^{\rho}}{\rho} d s \\
& +\sum_{i=1}^{n} \sum_{j=1}^{n} \int_{0}^{\infty} k_{j}(s)\left[\left|u_{j i}\right| F_{i}\left(\frac{1}{\delta_{3}^{\rho-1}}\right)+\left|v_{j i}\right| F_{i}\left(\frac{1}{\delta_{3}^{\rho-1}}\right)\right] \frac{\left|\xi_{i}(s-\tau)\right|^{\rho}}{\rho} d s \\
& \leq-\sum_{i=1}^{n}\left[\omega\left(d_{i}+\left|\phi_{i}(t)\right|+\left|\Psi_{i}(t)\right|\right)-\sum_{j=1}^{n}\left|c_{i j}\right| F_{j} \delta_{1}(\omega-1)\right. \\
& \left.-\sum_{j=1}^{n}\left|c_{j i}\right| F_{i} \frac{1}{\delta_{1}^{\omega-1}}-\sum_{j=1}^{n}\left|a_{i j}\right| F_{j} \delta_{2}(\omega-1)\right] \frac{\left|\xi_{i}(t)\right|^{\omega}}{\omega}+\sum_{i=1}^{n}\left[\sum_{j=1}^{n}\left|a_{j i}\right| F_{i} \frac{1}{\delta_{2}^{\omega-1}}\right] \frac{\left|\xi_{i}(t-\tau)\right|^{\omega}}{\omega} \\
& +\sum_{i=1}^{n} \sum_{j=1}^{n} \int_{0}^{\infty} k_{j}(s)\left[\left|u_{i j}\right| F_{j}(\omega-1) \delta_{3}+\left|v_{i j}\right| F_{j}(\omega-1) \delta_{3}\right] \frac{\left|\xi_{i}(s)\right|^{\omega}}{\omega} d s \\
& +\sum_{i=1}^{n} \sum_{j=1}^{n} \int_{0}^{\infty} k_{j}(s)\left[\left|u_{j i}\right| F_{i}\left(\frac{1}{\delta_{3}^{\omega-1}}\right)+\left|v_{j i}\right| F_{i}\left(\frac{1}{\delta_{3}^{\omega-1}}\right)\right] \frac{\left|\xi_{i}(s-\tau)\right|^{\omega}}{\omega} d s
\end{aligned}
$$




$$
\begin{aligned}
& -\sum_{i=1}^{n}\left[\rho\left(d_{i}+\left|\phi_{i}(t)\right|+\left|\Psi_{i}(t)\right|\right)-\sum_{j=1}^{n}\left|c_{i j}\right| F_{j} \delta_{1}(\rho-1)\right. \\
& \left.-\sum_{j=1}^{n}\left|c_{j i}\right| F_{i} \frac{1}{\delta_{1}^{\rho-1}}-\sum_{j=1}^{n}\left|a_{i j}\right| F_{j} \delta_{2}(\rho-1)\right] \frac{\left|\xi_{i}(t)\right|^{\rho}}{\rho}+\sum_{i=1}^{n}\left[\sum_{j=1}^{n}\left|a_{j i}\right| F_{i} \frac{1}{\delta_{2}^{\rho-1}}\right] \frac{\left.\xi_{i}(t-\tau)\right|^{\rho}}{\rho} \\
& +\sum_{i=1}^{n} \sum_{j=1}^{n} \int_{0}^{\infty} k_{j}(s)\left[\left|u_{i j}\right| F_{j}(\rho-1) \delta_{3}+\left|v_{i j}\right| F_{j}(\rho-1) \delta_{3}\right] \frac{\left|\xi_{i}(s)\right|^{\rho}}{\rho} d s \\
& +\sum_{i=1}^{n} \sum_{j=1}^{n} \int_{0}^{\infty} k_{j}(s)\left[\left|u_{j i}\right| F_{i}\left(\frac{1}{\delta_{3}^{\rho-1}}\right)+\left|v_{j i}\right| F_{i}\left(\frac{1}{\delta_{3}^{\rho-1}}\right)\right] \frac{\left|\xi_{i}(s-\tau)\right|^{\rho}}{\rho} d s \\
& \leq-\theta_{1}(t) V(t, \xi(t))+\theta_{2} \max _{t-\tau \leq s \leq t} V(s, \xi(s)) .
\end{aligned}
$$

where

$$
\begin{aligned}
\theta_{1}(t) & =\min _{1 \leq i \leq n}\left[\omega\left(d_{i}+\left|\phi_{i}(t)\right|+\left|\Psi_{i}(t)\right|\right)-\sum_{j=1}^{n}\left|c_{i j}\right| F_{j} \delta_{1}(\omega-1)-\sum_{j=1}^{n}\left|c_{j i}\right| F_{i} \frac{1}{\delta_{1}^{\omega-1}}\right. \\
& \left.-\sum_{j=1}^{n}\left|a_{i j}\right| F_{j}(\omega-1) \delta_{2}+\left|u_{i j}\right| F_{j}(\omega-1) \delta_{3}+\left|v_{i j}\right| F_{j}(\omega-1) \delta_{3}\right] \frac{\left|\xi_{i}(t)\right|^{\omega}}{\omega} \\
& +\min _{1 \leq i \leq n}\left[\rho\left(d_{i}+\left|\phi_{i}(t)\right|+\left|\Psi_{i}(t)\right|\right)-\sum_{j=1}^{n}\left|c_{i j}\right| F_{j} \delta_{1}(\rho-1)-\sum_{j=1}^{n}\left|c_{j i}\right| F_{i} \frac{1}{\delta_{1}^{\rho-1}}\right. \\
& \left.-\sum_{j=1}^{n}\left|a_{i j}\right| F_{j}(\rho-1) \delta_{2}+\left|u_{i j}\right| F_{j}(\rho-1) \delta_{3}+\left|v_{i j}\right| F_{j}(\rho-1) \delta_{3}\right] \frac{\left|\xi_{i}(t)\right|^{\rho}}{\rho} \\
\theta_{2}(t) & =\max _{1 \leq i \leq n}\left[\sum_{j=1}^{n}\left|a_{j i}\right| F_{i} \frac{1}{\delta_{2}^{\omega-1}}+\sum_{j=1}^{n}\left|u_{j i}\right| F_{i} \frac{1}{\delta_{3}^{\omega-1}}+\sum_{j=1}^{n}\left|v_{j i}\right| F_{i} \frac{1}{\delta_{3}^{\omega-1}} \frac{\left|\xi_{i}(t-\tau)\right|^{\omega}}{\omega}\right. \\
& +\max _{1 \leq i \leq n}\left[\sum_{j=1}^{n}\left|a_{j i}\right| F_{i} \frac{1}{\delta_{2}^{\rho-1}}+\sum_{j=1}^{n}\left|u_{j i}\right| F_{i} \frac{1}{\delta_{3}^{\rho-1}}+\sum_{j=1}^{n}\left|v_{j i}\right| F_{i} \frac{1}{\delta_{3}^{\rho-1}} \frac{\left|\xi_{i}(t-\tau)\right|^{\rho}}{\rho}\right.
\end{aligned}
$$

From this estimate, for any solution (13), which satisfies the Razumichin condition

$$
V(s, \xi(s)) \leq V(t, \xi(t)), t-\tau \leq s \leq t,
$$

we have

$$
{ }^{C} D^{\alpha} V(t, \xi(t)) \leq-\left(\eta_{1}-\eta_{2}\right) V(t, \xi(t)), t \geq 0 .
$$

According to Lemma 5, the claim of Theorem 4.4 follows.

$$
V(t, \xi(t)) \leq V(0, \xi(0)) E_{\alpha}\left(-\gamma t^{\alpha}\right), t \geq 0
$$

By Lemma 4 and inequality (37), this proves the system (10) can be achieved globally Mittag-Leffler stabilization under the designed control law. Hence complete the proof.

Remark 4.6. In the FCNNs, the fuzzy minimum feed-forward refers to taking the minimum value of the connection parameters with the fuzzy AND operation, and the fuzzy maximum feed-forward refers to taking the maximum value of the connection parameters with the fuzzy OR operation. The fuzzy minimum feedback refers to taking the minimum value of the delay connection parameter with the fuzzy $A N D$ operation, and the fuzzy maximum feedback refers to the maximum value of the delay connection parameter with the fuzzy OR operation. There are many other fuzzy logics, such as fuzzy AND, fuzzy OR, fuzzy NOT, fuzzy NAND, fuzzy NOR, fuzzy XOR, fuzzy XNOR, complex fuzzy logic and etc. It is worth highlighting that our work can be applied to FCNNs with more complex fuzzy logic. 
Remark 4.7. Neural networks usually have a spatial extension due to the presence of a multitude of parallel pathways with a variety of axon sizes and length and hence there is a distribution of propagation delays over a period of time. It is worth noting that although the signal propagation is sometimes instantaneous and can be modelled with discrete delays, it may also be distributed during a certain time period, so the distributed delays should be incorporated in the model. In other words, it is often the case that the neural network model possesses both discrete and distributed delays. In recent years, the stability of Hopfield neural networks, cellular neural networks and bidirectional associative memory neural networks with distributed delays has been discussed (see [40, 59] and refernces threin).

Remark 4.8. In recent days, stability analysis of fuzzy cellular neural networks have been extensively studied by te researchers. Stability analysis of fuzzy cellular neural networks with time delay in the leakage term and impulsive perturbations investigated in [79]. Finite-time synchronization of delayed fuzzy cellular neural networks with discontinuous activations is studied (see [80] and refernces there in). In these works integer order is considered in our paper fractional order is consideered. Great efforts have been made in study of fractional order neural networks bythe researchers. See for exxample, Adaptive control for fractional order induced chaotic fuzzy cellular neural networks [81], asymptotic stability of delayed fractional-order fuzzy neural networks with impulse effects [82], Mittag-Leffler stability of fractional-order Hopfield neural networks [83], Synchronization of fractional fuzzy cellular neural networks with interactions [84] have been invesitgated. In our paper stability fractional order fuzzy cellular neural networks with hybrid control is investigated.

Remark 4.9. In [85], Xu et al. investigated complete synchronization of fractional order complex networks via pinning adaptive and impulsive control strategy. In the past few years, due to the limited theory to deal with finite time stability of fractional order systems, synchronization research of fractional order systems is only the beginning [86] - [87]. In [88], Velmurugan et al. studied synchronization of fractional order neural networks by employing linear feedback control strategy. In [89], Ding et al. designed a novel controller based on feedback control strategy and discontinuous control strategy, and investigated stability of the considered fractional order neural networks. As is known to all, it is more perfect if the designed controller both realize the network synchronization objective and reduce the corresponding control cost. Motivated by the above discussions, we designed a novel hybrid feedback controller, which is based on a simple discontinuous control strategy and study stability problem of fractional order cellular neural networks networks.

Remark 4.10. It is well known that every control strategy has its own advantages and disadvantages. Therefore, in some cases, hybrid control strategies are necessary to control some complex systems to achieve synchronization or other properties. Since fuzzy cellular neural networks may experience instantaneous perturbation or abrupt changes at certain instances, hybrid impulsive and other control strategies could be used to control them to the synchronous state. However, to authors' knowledge, there have few research achievements on the stability of fuzzy cellular neural networks by means of hybrid control strategies at present. It is still an open problem that deserves further investigation, which motivated our research in this paper.

\section{Numerical EXAmple}

In this section, two examples are given to illustrate the effectiveness of proposed Global MittagLeffler stability schemes.

Example 1: For $n=4$, consider the following fractional-order fuzzy cellular neural networks,

$$
\begin{aligned}
{ }^{C} D^{\alpha} \zeta_{i}(t) & =-d_{i} \zeta_{i}(t)+\sum_{j=1}^{2} c_{i j} f_{j}\left(\zeta_{j}(t)\right)+\sum_{j=1}^{2} a_{i j} f_{j}\left(\zeta_{j}(t-\tau)+\sum_{j=1}^{2} h_{i j} \mu_{j}\right. \\
& +\bigwedge_{j=1}^{2} u_{i j} \int_{0}^{\infty} k_{j}(s) f_{j}\left(\zeta_{j}(s-\tau)\right) d s+\bigwedge_{j=1}^{2} p_{i j} \mu_{j}+\bigvee_{j=1}^{n} v_{i j} \int_{0}^{\infty} k_{j}(s) f_{j}\left(\zeta_{j}(s-\tau)\right) d s
\end{aligned}
$$




$$
+\bigvee_{j=1}^{2} Q_{i j} \mu_{j}+I_{i}, i=1,2,
$$

The parameters of (13) error system are assumed that $a_{11}=0.4, a_{12}=0.6, a_{13}=0.9, a_{14}=1.2$, $a_{21}=0.8, a_{22}=0.9, a_{23}=2.3, a_{24}=1.5, a_{31}=0.7, a_{32}=1.8, a_{33}=0.5, a_{34}=0.7 a_{41}=1.3$, $a_{42}=0.5, a_{43}=0.2, a_{44}=0.9, c_{11}=1.5, c_{12}=1.8, c_{13}=0.7, c_{14}=1.3 c_{21}=2.9, c_{22}=2.8, c_{23}=0.9$ $c_{24}=0.7 c_{31}=1.8, c_{32}=1.5, c_{33}=0.7, c_{34}=0.9 c_{41}=2.8, c_{42}=2.9, c_{43}=0.7, c_{44}=1.3, u_{11}=0.9$, $u_{12}=1.2, u_{13}=2.1, u_{14}=3.4, u_{21}=2.1, u_{22}=3.4, u_{23}=1.2, u_{24}=0.9, u_{31}=2.1, u_{32}=1.2$, $u_{33}=0.9, c_{34}=1.7 u_{41}=1.7, u_{42}=1.7, u_{43}=1.2, u_{44}=0.9, v_{11}=1.8, v_{12}=2.2, v_{13}=0.7$, $v_{14}=2.7, v_{21}=2.2, v_{22}=0.9, v_{23}=0.5, v_{24}=1.2, v_{31}=1.7, v_{32}=2.2, v_{33}=0.7, v_{34}=0.9$ $v_{41}=0.9, v_{42}=0.5, v_{43}=1.8, v_{44}=1.7, d_{1}=5, d_{2}=7, d_{3}=7, d_{4}=5, \Psi_{1}=0.6, \Psi_{2}=0.7$, $\Psi_{3}=0.5, \Psi_{4}=0.4, \phi_{1}=1.4, \phi_{2}=3.1, \phi_{3}=2.7, \phi_{4}=3.7, \alpha=0.97, f(t)=\tanh (t)$.

The corresponding response system is described by

$$
\begin{aligned}
{ }^{C} D^{\alpha} \vartheta_{i}(t) & =-d_{i} \vartheta_{i}(t)+\sum_{j=1}^{2} c_{i j} f_{j}\left(\vartheta_{j}(t)\right)+\sum_{j=1}^{2} a_{i j} f_{j}\left(\vartheta_{j}(t-\tau)+\sum_{j=1}^{2} h_{i j} \mu_{j}\right. \\
& +\bigwedge_{j=1}^{2} u_{i j} \int_{0}^{\infty} k_{j}(s) f_{j}\left(\vartheta_{j}(s-\tau)\right) d s+\bigwedge_{j=1}^{2} p_{i j} \mu_{j}+\bigvee_{j=1}^{2} v_{i j} \int_{0}^{\infty} k_{j}(s) f_{j}\left(\vartheta_{j}(s-\tau)\right) d s \\
& +\bigvee_{j=1}^{2} Q_{i j} \mu_{j}+I_{i}+u_{i}(t),
\end{aligned}
$$

where $a_{i j}, u_{i j}, v_{i j}, d_{i}, c_{i j}$, and $I_{i}$ are the same as in system (39), and the hybrid feedback controllers is as follows: $u_{i}(t)=u_{1 i}(t)+u_{2 i}(t)$, where $u_{1 i}(t)=-\phi_{i}(t) \xi_{i}(t)$ and $u_{2 i}(t)=-\Psi_{i}(t) \xi_{i}(t)$. where $\xi_{i}(t)=\vartheta_{i}(t)-\zeta_{i}(t)$ for $\mathrm{i}=1,2$. According to the developed idea in Theorem 4.1, it asks that

$$
\begin{aligned}
\eta_{1}(t) & =\min _{1 \leq i \leq n}\left[\left(d_{i}+\phi_{i}(t)+\Psi_{i}\right)-\sum_{j=1}^{n} c_{i j} F_{j} \rho^{-1}-\sum_{j=1}^{n} c_{j i} F_{i} \rho-\sum_{j=1}^{n} a_{i j} F_{j} \rho^{-1}\right. \\
& \left.+\sum_{j=1}^{n} u_{i j} F_{j} \rho^{-1}+\sum_{j=1}^{n} v_{i j} F_{j} \rho^{-1}\right]=12.33>0, \\
\eta_{2}(t) & =\max _{1 \leq i \leq n}\left[\sum_{j=1}^{n} a_{j i} F_{i} \rho+u_{j i} F_{i} \rho+\sum_{j=1}^{n} v_{j i} F_{i} \rho\right]=12.18>0 .
\end{aligned}
$$

Then it follows from Theorem 4.1 that system (38) can be achieved global Mittag-Leffler stabilization under the designed hybrid feedback control law.

Example 2: For $\mathrm{n}=2$, consider the following fractional-order fuzzy cellular neural networks,

$$
\begin{aligned}
{ }^{C} D^{\alpha} \zeta_{i}(t) & =-d_{i} \zeta_{i}(t)+\sum_{j=1}^{2} c_{i j} f_{j}\left(\zeta_{j}(t)\right)+\sum_{j=1}^{2} a_{i j} f_{j}\left(\zeta_{j}(t-\tau)+\sum_{j=1}^{2} h_{i j} \mu_{j}\right. \\
& +\bigwedge_{j=1}^{2} u_{i j} \int_{0}^{\infty} k_{j}(s) f_{j}\left(\zeta_{j}(s-\tau)\right) d s+\bigwedge_{j=1}^{2} p_{i j} \mu_{j}+\bigvee_{j=1}^{n} v_{i j} \int_{0}^{\infty} k_{j}(s) f_{j}\left(\zeta_{j}(s-\tau)\right) d s \\
& +\bigvee_{j=1}^{2} Q_{i j} \mu_{j}+I_{i}, i=1,2,
\end{aligned}
$$

where $\alpha=0.97, f(t)=\tanh (t)$

The parameters of (13) error system are assumed that $a_{11}=0.9, a_{12}=0.8, a_{13}=0.4, a_{14}=0.6$, $a_{21}=0.4, a_{22}=0.8, a_{23}=0.7, a_{24}=0.3, a_{31}=0.3, a_{32}=0.7, a_{33}=0.7, a_{34}=0.5 a_{41}=0.5$, $a_{42}=0.8, a_{43}=0.3, a_{44}=0.5, c_{11}=0.4, c_{12}=0.5, c_{13}=0.4, c_{14}=1.3 c_{21}=0.7, c_{22}=0.8, c_{23}=0.5$ 


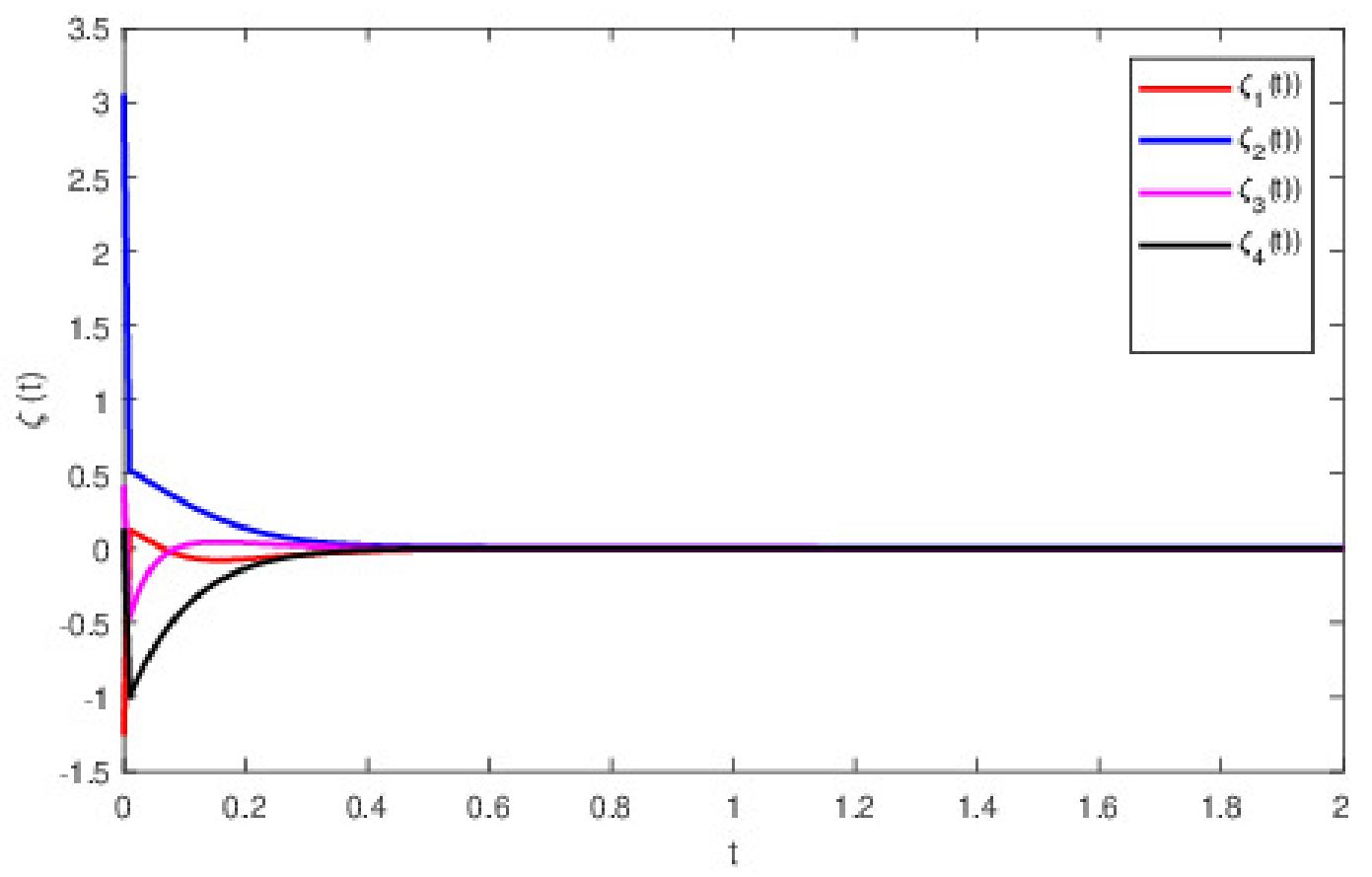

FIgURE 1. State trajectories of the FCNN system in Example 1

$c_{24}=0.7 c_{31}=0.4, c_{32}=0.7, c_{33}=0.9, c_{34}=0.3 c_{41}=0.3, c_{42}=0.8, c_{43}=0.5, c_{44}=0.7, u_{11}=0.9$, $u_{12}=1.1, u_{13}=1.3, u_{14}=0.7, u_{21}=1.3, u_{22}=0.9, u_{23}=0.8, u_{24}=1.2, u_{31}=1.2, u_{32}=0.7$, $u_{33}=1.1, u_{34}=1 u_{41}=1.3, u_{42}=1, u_{43}=0.5, u_{44}=0.3, v_{11}=0.7, v_{12}=1.1, v_{13}=1, v_{14}=1.3$, $v_{21}=1, v_{22}=0.3, v_{23}=0.7, v_{24}=0.2, v_{31}=0.4, v_{32}=0.8, v_{33}=0.9, v_{34}=1.2 v_{41}=1.1, v_{42}=0.2$, $v_{43}=0.7, v_{44}=1.2, d_{1}=9, d_{2}=9, d_{3}=7, d_{4}=7, \Psi_{1}=2.7, \Psi_{2}=3.1, \Psi_{3}=4.5, \Psi_{4}=2.5, \phi_{1}=5.3$, $\phi_{2}=4.7, \phi_{3}=5.3, \phi_{4}=5.1, \alpha=0.97, f(t)=\tanh (t)$.

$\omega=2, \delta_{1}=\delta_{2}=\delta_{3}=1, F_{i}=1,(i=1,2,3,4)$.

The corresponding response system is described by

$$
\begin{aligned}
{ }^{C} D^{\alpha} \vartheta_{i}(t) & =-d_{i} \vartheta_{i}(t)+\sum_{j=1}^{2} c_{i j} f_{j}\left(\vartheta_{j}(t)\right)+\sum_{j=1}^{2} a_{i j} f_{j}\left(\vartheta_{j}(t-\tau)+\sum_{j=1}^{2} h_{i j} \mu_{j}\right. \\
& +\bigwedge_{j=1}^{2} u_{i j} \int_{0}^{\infty} k_{j}(s) f_{j}\left(\vartheta_{j}(s-\tau)\right) d s+\bigwedge_{j=1}^{2} p_{i j} \mu_{j}+\bigvee_{j=1}^{2} v_{i j} \int_{0}^{\infty} k_{j}(s) f_{j}\left(\vartheta_{j}(s-\tau)\right) d s \\
& +\bigvee_{j=1}^{2} Q_{i j} \mu_{j}+I_{i}+u_{i}(t),
\end{aligned}
$$

where $a_{i j}, u_{i j}, v_{i j}, d_{i}, c_{i j}$, and $I_{i}$ are the same as in system (41), and the hybrid feedback controllers is as follows: $u_{i}(t)=u_{1 i}(t)+u_{2 i}(t)$, where $u_{1 i}(t)=-\phi_{i}(t) e_{i}(t)$ and $u_{2 i}(t)=-\Psi_{i}(t) e_{i}(t)$. where $\xi_{i}(t)=\vartheta_{i}(t)-\zeta_{i}(t)$ for $\mathrm{i}=1,2$. According to the developed idea in Theorem 4.2, it asks that

$$
\theta_{1}(t)=\min _{1 \leq i \leq n}\left[\left(d_{i}+\left|\phi_{i}(t)\right|+\left|\Psi_{i}(t)\right|\right)-\sum_{j=1}^{n}\left|c_{i j}\right| F_{j} \delta_{1}(\omega-1)-\sum_{j=1}^{n}\left|c_{j i}\right| F_{i} \frac{1}{\delta_{1}^{\omega-1}}\right.
$$




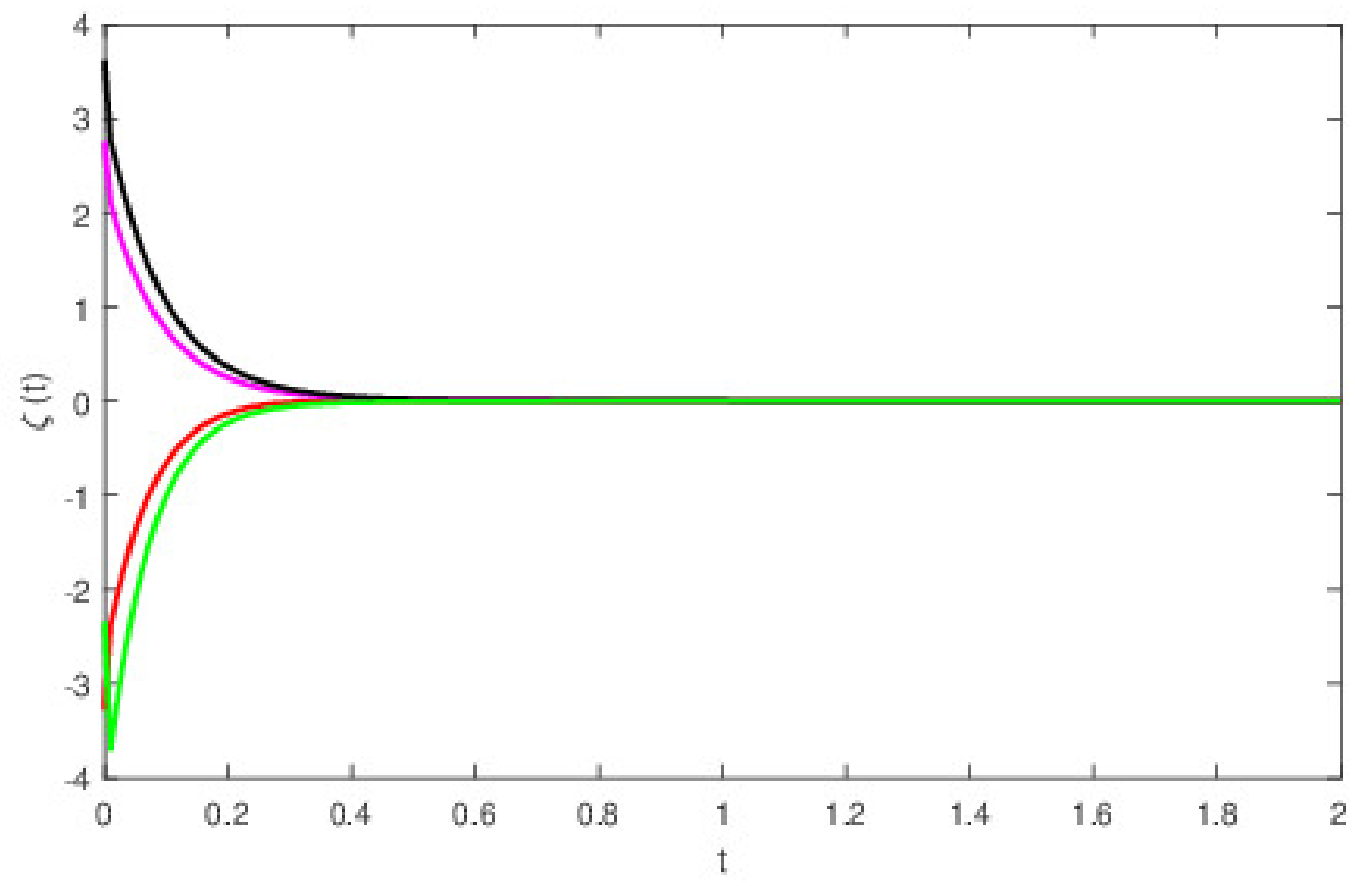

FIgURE 2. State trajectories of the FCNN system in Example 2

$$
\begin{array}{r}
\left.+\sum_{j=1}^{n}\left|a_{i j}\right| F_{j}(\omega-1) \delta_{2}+\left|u_{i j}\right| F_{j}(\omega-1) \delta_{3}+\left|v_{i j}\right| F_{j}(\omega-1) \delta_{3}\right]=10.40>0 \\
\theta_{2}(t)=\max _{1 \leq i \leq n}\left[\sum_{j=1}^{n}\left|a_{j i}\right| F_{i} \frac{1}{\delta_{2}^{\omega-1}}+\sum_{j=1}^{n}\left|u_{j i}\right| F_{i} \frac{1}{\delta_{3}^{\omega-1}}+\sum_{j=1}^{n}\left|v_{j i}\right| F_{i} \frac{1}{\delta_{3}^{\omega-1}}\right]=10>0 .
\end{array}
$$

Then it follows from Theorem 4.2 that system (40) can be achieved global Mittag-Leffler stabilization under the designed hybrid feedback control law.

\section{Conclusion}

In this paper, Global Mittag-Leffler stability of fractional order fuzzy cellular neural networks with distributed delays via hybrid feedback controllers is addressed. We proposed the definition of Global Mittag-Leffler stability and the fractional Lyapunov direct method, which enriches the knowledge of both the system theory and the fractional calculus. This papers serves as a first step in presenting sufficient conditions for global Mittag-Leffler stability for a generalized fuzzy cellular fractional-order neural network with distributed delay. The main theoretical findings of this paper are that hybrid feedback control law. We extend the concept of Lyapunov functions for a fuzzy cellular fractionalorder neural network. Under the assumption of Lipschitz continuity of activation functions, we have proved the Global Mittag-Leffler stability of the proposed model, which implies faster convergence rate of the network model than the Lyapunov convergence. An illustrative examples was provided to demonstrate the applicability of the proposed approach. 


\section{REFERENCES}

[1] E. S. A. Shahri, S. Balochian, Analysis of fractional-order linear systems with saturation using Lyapunov's second method and convex optimization, International Journal of Automation and Computing 12(4)(2015)440-447.

[2] P. Arena, R. Caponetto, L. Fortuna, D. Porto, Bifurcation and chaos in non integer order cellular neural networks, International Journal of Bifurcation and Chaos, 8(1998)1527 - 1539.

[3] M. Syed Ali, L Palanisamy, J Yogambigai, L Wang, Passivity-based synchronization of Markovian jump complex dynamical networks with time-varying delays, parameter uncertainties, reaction-diffusion terms, and sampled-data control Journal of Computational and Applied Mathematics 352 (2019) 79-92.

[4] M. Syed Ali, J Yogambigai, S Saravanan, S Elakkia, Stochastic stability of neutral-type Markovian-jumping BAM neural networks with time varying delays, Journal of Computational and Applied Mathematics 349 (2019) 142-156

[5] M. Syed Ali, J Yogambigai Extended dissipative synchronization of complex dynamical networks with additive time-varying delay and discrete-time information Journal of Computational and Applied Mathematics 348 (2019) 328-341

[6] I. Stamova, Global Mittag-Leffler stability and synchronization of impulsive fractional-order neural networks with time-varying delays, Nonlinear Dynamics, 77 (2014) 1251 - 1260.

[7] H. Q. Li, X. F. Liao, and M. W. Luo, A novel non-equilibrium fractional-order chaotic system and its complete synchronization by circuit implementation, Nonlinear Dynamics, 68 (2012) 137 - 149.

[8] L. P. Chen, Y. Chai, R. C. Wu, T. D. Ma, and H. Z. Zhai, Dynamic analysis of a class of fractional-order neural networks with delay, Neurocomputing, 111 (2013) 190 - 194.

[9] Z, Quanxin, S. Shiyun, T. Tianren, Mean square exponential stability of stochastic nonlinear delay systems of International Journal of Control, 90 (2017) $2384-2393$.

[10] X. Li, X. Yang, T. Huang, Persistence of delayed cooperative models, Impulsive control method, Applied Mathematics and Computation, 342 (2019) 130-146.

[11] X. Li, J. Shen, R. Rakkiyappan, Persistent impulsive effects on stability of functional differential equations with finite or infinite delay, Applied Mathematics and Computation, 329 (2018) 14-22

[12] M.Syed Ali, G.Narayanana, S. Sevgen, V. Shekher, S. Arik, Global stability analysis of fractional-order fuzzy BAM neural networks with time delay and impulsive effects, Communications in Nonlinear Science and Numerical Simulation, 78 (2019) Article ID 104853.

[13] E. Yucel, M.Syed Ali, N. Gunasekaran, S. Arik, Sampled-data filtering of Takagi-Sugeno fuzzy neural networks with interval time-varying delays, Fuzzy Sets and Systems, 316 (2017) 69-81.

[14] N. Ozcan, M.Syed Ali, J. Yogambigai, Q. Zhu, S. Arik, Robust synchronization of uncertain Markovian jump complex dynamical networks with time-varying delays and reaction-diffusion terms via sampled-data control, Journal of the Franklin Institute, $\mathbf{3 5 5} 3$ (2018) 1192-1216.

[15] C. Song, S. Fei, J. Cao, C. Huang, Robust synchronization of fractional-order uncertain chaotic systems based on output feedback sliding mode control, Mathematics 7(7) (2019) 599.

[16] X. Yang, S. Wen, Z. Liu, C. Li, C. Huang, Dynamic Properties of Foreign Exchange Complex Network, Mathematics 7 (2019) 832.

[17] F. Wang, Z. Yao, Approximate controllability of fractional neutral differential systems with bounded delay. Fixed Point Theory. 17 (2016) 495-507.

[18] G. Rajchakit, A. Pratap, R.Raja, J.Cao, J. Alzabut, C. Huang, Hybrid control scheme for projective lag synchronization of Riemann-Liouville sense fractional order memristive BAM neural networks with mixed delays. Mathematics $\mathbf{7 ( 8 )}$ (2019) 759 .

[19] X. Yang, S. Wen, Z. Liu, C. Li, C. Huang, Dynamic properties of foreign exchange complex network, Mathematics, 7 (2019) 832.

[20] C. Huang, S. Wen, L. Huang, Dynamics of anti-periodic solutions on shunting inhibitory cellular neural networks with multi-proportional delays, Neurocomputing 357 (2019) 47-52.

[21] J. Zhang, C. Huang, Dynamics analysis on a class of delayed neural networks involving inertial terms, Advances in Difference Equations (2020) Article 120.

[22] C. Qian, Y. Hu ,Novel stability criteria on nonlinear density-dependent mortality Nicholson's blowflies systems in asymptotically almost periodic environments, Journal of Inequalities and Applications (2020) Article number: 13.

[23] J. Zhang, C. Huang, Dynamics analysis on a class of delayed neural networks involving inertial terms, Advances in Difference Equations (2020) Article number: 120.

[24] M. Shi, J. Guo, X. Fang, C. Huang, Global exponential stability of delayed inertial competitive neural networks, Advances in Difference Equations (2020) Article number: 87.

[25] Q. Cao,G. Wang, C. Qian, New results on global exponential stability for a periodic Nicholson's blowflies model involving time-varying delays, Advances in Difference Equations (2020) Article number: 43.

[26] C. Huang ,R. Su, Y. Hu, Global convergence dynamics of almost periodic delay Nicholson's blowflies systems , Journal of Biological Dynamics 14 (2020) 633-655.

[27] J. Wang, X. Chen, L. Huang, The number and stability of limit cycles for planar piecewise linear systems of node-saddle type, Journal of Mathematical Analysis and Applications, 469 (1) (2019) 405-427. 
[28] J. Wang, C. Huang, L. Huang, Discontinuity-induced limit cycles in a general planar piecewise linear system of saddle-focus type, Nonlinear Analysis: Hybrid Systems, 33 (2019) 162-178.

[29] W. Wang, Finite-time synchronization for a class of fuzzy cellular neural networks with time-varying coefficients and proportional delays, Fuzzy Sets and Systems, 338 (2018) 40-49.

[30] C. Huang and H. Zhang, Periodicity of non-autonomous inertial neural networks involving proportional delays and non-reduced order method, International Journal of Biomathematics,12(2) (2019) 1950016.

[31] H. Yang, Weighted pseudo almost periodicity on neutral type CNNs involving multi-proportional delays and D operator, AIMS Mathematics (6) (2021) 1865-1879.

[32] C. Huang, L. Yang, J. Cao Asymptotic behavior for a class of population dynamics, AIMS Mathematics, 5 (2020) $3378-3390$

[33] C. Huang, X. Long,L. Huang and Si Fu, Stability of Almost Periodic Nicholson's Blowflies Model Involving Patch Structure and Mortality Terms Canadian Mathematical Bulletin , 63 (2) (2020) 405 - 422.

[34] C. Huang, J. Wang, L. Huang, Asymptotically almost periodicity of delayed Nicholson-type system involving patch structure, Electron. J. Differential Equations61 (2020) 1-17.

[35] H. Hu, T. Yi and X. Zou., On spatial-temporal dynamics of a Fisher-KPP equation with a shifting environment, Proc. Amer. Math. Soc. 148 (2020) 213-221.

[36] HJ Hu, XP Yuan, LH Huang, CX Huang, Global dynamics of an SIRS model with demographics and transfer from infectious to susceptible on heterogeneous networks. Math. Biosci. Eng. 16(5) (2019) 5729-5749.

[37] H. Hu and X. Zou. Existence of an extinction wave in the Fisher equation with a shifting habitat Proc. Amer. Math. Soc. 145 (2017) 4763-4771.

[38] SM. Lee, OM. Kwon, SH. Lee, Improved stability criteria for sampled-data systems using modified free weighting matrix Journal of the Franklin Institute, 356 (2019) 2198 - 2211.

[39] P. Balasubramaniam, M. Syed Ali Robust stability of uncertain fuzzy cellular neural networks with time-varying delays and reaction diffusion terms, Neurocomputing, $\mathbf{7 4}(2010) 439-446$.

[40] X. Li and S. Song, Impulsive control for existence, uniqueness and global stability of periodic solutions of recurrent neural networks with discrete and continuously distributed delays, IEEE Transactions on Neural Networks and Learning Systems, 24 (2013) 868 - 877.

[41] Y. Du, S. Zhong, N. Zhou, K. Shi, J. Cheng, Exponential stability for stochastic Cohen-Grossberg BAM neural networks with discrete and distributed time-varying delays, Neurocomputing, 127 (2014) $144-151$.

[42] M. Syed Ali, N. Gunasekaran, ME. Rani, Robust stability of hopfield delayed neural networks via an augmented LK functional, Neurocomputing, 234 (2017) 198 - 204.

[43] S. Liu, XY. Li, W. Jiang, XF. Zhou, Mittag-Leffler stability of nonlinear fractional neutral singular systems, Communications in Nonlinear Science and Numerical Simulation, 17(2012)3961 - 3966.

[44] Y. Li, Y.Q. Chen, I. Podlubny, Stability of fractional-order nonlinear dynamic systems: Lyapunov direct method and generalized Mittag-Leffler stability, Computers and Mathematics with Applications, 24 (2010) 1429 - 1468

[45] L. O. Chua and L. Yang, Cellular neural networks: Theory, IEEE Transactions on Circuits and Systems, 35 (1988) $1257-1272$.

[46] T. Roska and L. O. Chua, Cellular neural networks with nonlinear and delay-type template elements and nonuniform grids, International Journal of Circuit Theory and Applications, 20 (1992) 469 - 481.

[47] H. Harrer and J. A. Nossek, Discrete-time cellular neural networks, International Journal of Circuit Theory and Applications, 20 (1992)453-467.

[48] T. Yang, LB. Yang, CW. Wu, LO. Chua, Fuzzy cellular neural networks:theory, International Journal of Circuit Theory and Applications, (1996)181- 186.

[49] T. Yang, LB. Yang, CW. Wu, LO. Chua, Fuzzy cellular neural networks:applications Fourth IEEE International Workshop on Cellular Neural Networks and their Applications Proceedings, (1996)225 - 230

[50] Z. D. Huang, Almost periodic solutions for fuzzy cellular neural networks with multi-proportional delays, International Journal of Machine Learning and Cybernetics, 78(2016) 1323 -1331.

[51] S. T. Wang and M. Wang, A new detection algorithm based on fuzzy cellular neural networks for white blood cell detection, IEEE Transactions on Information Technology in Biomedicine, 10 (2006) 5 - 10.

[52] W. Y. Ma, C. P. Li, and Y. J. Wu, Impulsive synchronization of fractional Takagi-Sugeno fuzzy complex networks, Chaos, 26 (2016)084311.

[53] H. Shen, F. Li, H. Yan, H. R. Karimi and H. K. Lam, Finite-time event-triggered $H_{\infty}$ control for T-S fuzzy Markov jump systems, IEEE Transactions on Fuzzy Systems, 26 (2018)3122 - 3135.

[54] H. Shen,F. Li, Z. Wu, J. H. Park, Victor Sreeram. Fuzzy-model-based non-fragile control for nonlinear singularly perturbed systems with semi-Markov jump parameters, IEEE Transactions on Fuzzy Systems, 26 (2018)3428 3439.

[55] M S. Branicky, Introduction to Hybrid Systems Springer, (2005)91 - 116.

[56] A. S. Morse, Control Using Logic-Based Switching, Springer, (1997)1- 276.

[57] P. J. Antsaklis, Special issue on hybrid systems: Theory and applications - A brief introduction to the theory and applications of hybrid systems, Proceedings of the IEEE, $8 \mathbf{8}(2000) 879-887$. 
[58] X. Li, J. Shen, H. Akca, R. Rakkiyappan, LMI-based stability for singularly perturbed nonlinear impulsive differential systems with delays of small parameter, Applied Mathematics and Computation, 250 (2015) 798-804.

[59] X. Li, Donal O'Regan, H. Akca, Global exponential stabilization of impulsive neural networks with unbounded continuously distributed delays, IMA Journal of Applied Mathematics, 80(1) (2015) 85-99.

[60] X. Li, T. Caraballo, R. Rakkiyappan, Xiuping Han, On the stability of impulsive functional differential equations with infinite delays, Mathematical Methods in the Applied Sciences, 38(14) (2015) 3130-3140.

[61] Y. Wardi, M. Egerstedt, B. Lennartson, P. Tabuada Hybrid Systems, Nonlinear Analysis, (2019).

[62] M. Syed Ali, J. Yogambigai, Synchronization of complex dynamical networks with hybrid coupling delays on time scales by handling multitude Kronecker product terms, Applied Mathematics and Computation, 291 (2016) 244 258.

[63] A. Feuer, G. C. Goodwin, and M. Salgado, Potential benefits of hybrid control for linear time invariant plants, Proceedings of the 1997 American Control Conference, 5 (1997)2790 - 2794.

[64] N. H. McClamroch, C. Rui, I. Kolmanovsky, and M. Reyhanoglu, Hybrid closed loop systems: A nonlinear control perspective, Proceedings of the 36th IEEE Conference on Decision and Control, 1 (1997) $114-119$.

[65] A. J. van der Schaft and J. M. Schumacher, An Introduction to Hybrid Dynamical Systems, Springer, 251(1999)1 -174 .

[66] J. Lygeros, D. Godbole, and S. Sastry, Verified hybrid controllers for automated vehicles, IEEE Transactions on Automatic Control, 43 (1998)522 - 539.

[67] E. Frazzoli, Robust hybrid control for autonomous vehicle motion planning, Proceedings of the IEEE Conference on Decision and Control, 1(2000)821 - 826.

[68] E. Frazzoli, M. A. Dahleh, and E. Feron, A maneuver-based hybrid control architecture for autonomous vehicle motion planning, Information Technology for Dynamical Systems, (2005)299 - 323.

[69] A. Balluchi, P. Soueres, and A. Bicchi, Hybrid feedback control for path tracking with a bounded-curvature vehicle, Proceedings of the fourth International Workshop on Hybrid Systems, (2001)133 - 146.

[70] K. Diethelm, N J. Ford, Analysis of Fractional Differential Equations, Journal of Mathematical Analysis and Applications, 265 (2002)229 - 248.

[71] C. Li, F. Zhang, A survey on the stability of fractional differential equations, The European Physical Journal Special Topics, 193(2011) $27-47$.

[72] M. A. Duatte-Mermoud, N. Aguila-Camacho, J. A. Gallegos, R. Castro-Limares, Using general quadratic Lyapunov functions to prove Lyapunov uniform stability for fractional order systems, Communications in Nonlinear Science and Numerical Simulation, 22 (2015) $650-659$.

[73] S. H. Maboobi, M. Shahrokhi, H. N. Pishkenari, Observer-based control design for three well-known chaotic systems, Chaos Solitions and Fractals, 29(2006) 381 -392.

[74] A. Wu, Z. Zeng, Global Mittag-Leffler stabilization of fractional-order memristive neural networks, IEEE Transactions on Neural Networks and Learning Systems, 28 (2015)1 - 12.

[75] J. Chen, Z. Zeng, P. Jiang, Global Mittag-Leffler stability and synchronization of memristor-based fractional-order neural networks, Neural Network: the official journal of the International Neural Network Society 51 (2013) 1 8.

[76] J. Kuang, Applied inequalities, Shandong Science and Technology Press, (2004).

[77] J. Chen, Z. Zeng, P. Jiang, Global Mittag-Leffler stability and synchronization of memristor-based fractional-order neural networks, Neural Networks, $\mathbf{5 1}$ (2014) 1-8.

[78] I . Stamova, G. Stamov, Mittag-Leffler synchronization of fractional neural networks with time-varying delays and reaction-diffusion terms using impulsive and linear controllers, , Neural Networks, 96 (2017) 22-32.

[79] S. Long Q. Song, X. Wang D. Li, Stability analysis of fuzzy cellular neural networks with time delay in the leakage term and impulsive perturbations, Journal of the Franklin Institute , 349 (7) (2012) 2461-2479.

[80] L. Duan, H. Wei, L. Huang, Finite-time synchronization of delayed fuzzy cellular neural networks with discontinuous activations Fuzzy Sets and Systems 361 (2019) 56-70.

[81] P. Mani, R. Rakkiyappan, S. Lakshmanan, Y. H. Joo, Adaptive control for fractional order induced chaotic fuzzy cellular neural networks and its application to image encryption, Information Sciences, 491 (2019) 74-89.

[82] J. Chen, C. Li, X.Yang, Asymptotic stability of delayed fractional-order fuzzy neural networks with impulse effects, Journal of the Franklin Institute 355 (15) (2018) 7595-7608

[83] S. Zhang, Y. Yu, H . Wang, Mittag-Leffler stability of fractional-order Hopfield neural networks, Nonlinear Anal. Hybrid Syst. 16 (2015) 104-121.

[84] W. Ma, C. Li, Y. Wu, Y. Wu, Synchronization of fractional fuzzy cellular neural networks with interactions, Chaos 27 (2017) 103106.

[85] H. Li, C. Hu, Y. Jiang, Z. Wang, Z. Dong, Pinning adaptive and impulsive synchronization of fractional-order complex dynamical networks, Chaos Solitons Fract. 92 (2016) 142-149.

[86] L. Wang, Q. Song, Y. Liu, Z. Zhao, F. Alsaadi, Finite-time stability analysis of fractional-order complex-valued memristor-based neural networks with both leakage and time-varying delays, Neurocomputing 245 (2017) 86-101.

[87] J. Xiao, S. Zhong, Y. Li, F. Xu, Finite-time Mittag-Leffler synchronization of fractional-order memristive BAM neural networks with time delays, Neurocomputing 219 (2017) 431-439. 
[88] G. Velmurugan, R. Rakkiyappan, J. Cao, Finite-time synchronization of fractionalorder memristor-based neural networks with time delays, Neural Netw. 73 (2016) 36-46.

[89] Z. Ding, Z. Zeng, L. Wang, Robust finite-time stabilization of fractional-order neural networks with discontinuous and continuous activation functions under uncertainty, IEEE Trans. Neural Netw. Learn. Syst. 29 (2018) 14771490. 
Figures

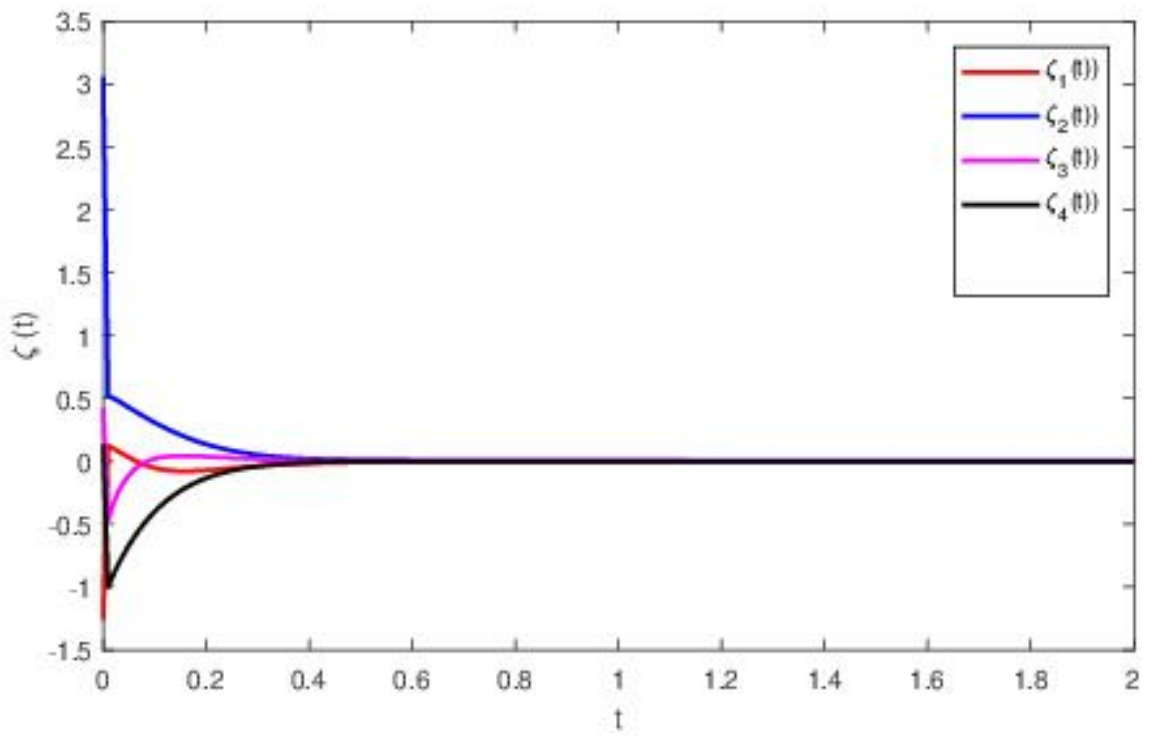

Figure 1

State trajectories of the FCNN system in Example 1

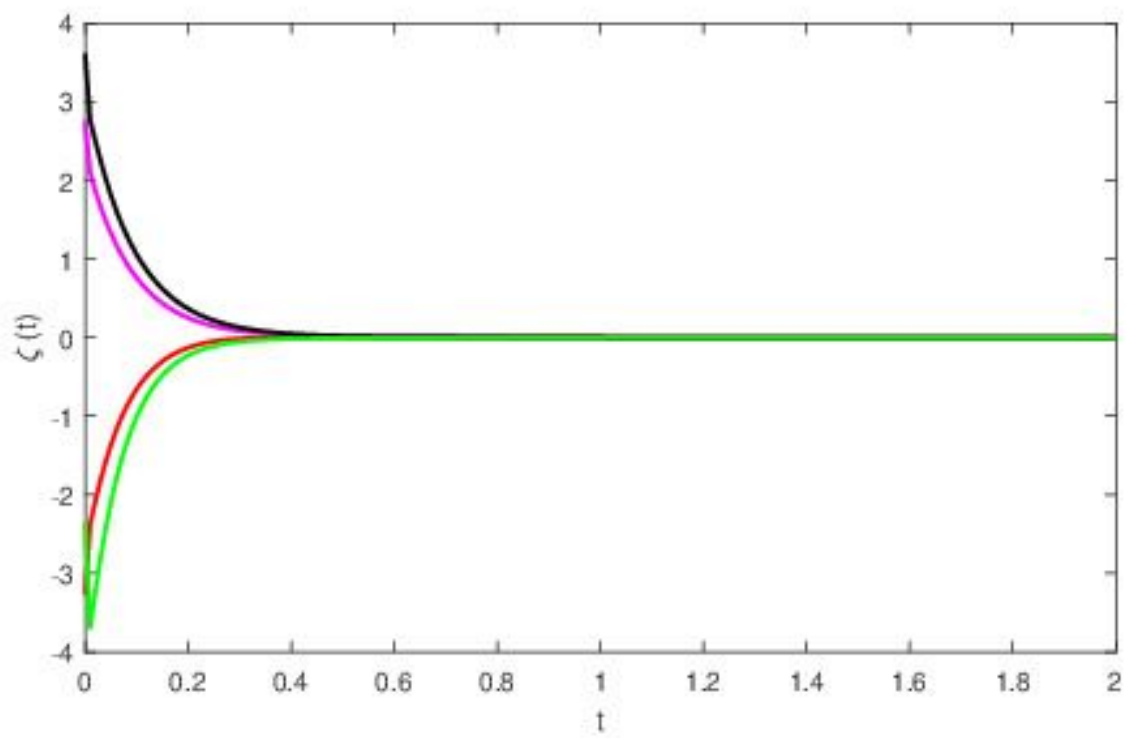

Figure 2

State trajectories of the FCNN system in Example 2 